The Astrophysical Journal, 482:1050-1064, 1997 June 20

(C) 1997. The American Astronomical Society. All rights reserved. Printed in U.S.A.

\title{
RELATIVE ELEMENTAL ABUNDANCES OF THE QUIET SOLAR CORONA AS DETERMINED BY SERTS
}

\author{
D. A. FALCONER ${ }^{1}$ \\ Physics Department, University of Maryland \\ AND \\ J. M. DAVILA AND R. J. THOMAS \\ Laboratory for Astronomy and Solar Physics, NASA/Goddard Space Flight Center, Code 682, Greenbelt, MD 20771 \\ Received 1995 November 13; accepted 1997 January 9
}

\begin{abstract}
Intensities of extreme-ultraviolet (EUV) spectral lines were measured as a function of radius off the solar limb by two flights (1989 May 5 and 1991 May 7) of the Solar Extreme-ultraviolet Rocket Telescope and Spectrograph (SERTS) for three quiet solar regions. The line-ratio density, line-ratio temperature, and emission measure were determined. The relative abundances of silicon, aluminum, and chromium to iron were determined. Results agreed with standard coronal relative elemental abundances for one observation, but did not agree for the other, in which aluminum was overabundant.
\end{abstract}

Subject headings: Sun: abundances - Sun: corona - UV radiation

\section{CORONAL ELEMENTAL ABUNDANCE}

For over a decade, there have been clear indications that the elemental composition of the corona differs from that of the photosphere (see, e.g., Veck \& Parkinson 1981). Such differences have been determined both form in situ solar wind and solar energetic particle measurements (Reames, Cane, \& von Rosenvinge 1990) and from spectroscopy (for recent reviews see Meyer 1993 and Stone 1989). Meyer (1985b) showed that the elemental abundance variation seems to depend most strongly on the first ionization potential (FIP) of the element. From solar wind measurements, the ratios of abundance of low-FIP (less than $10 \mathrm{eV}$ ) elements to high-FIP (greater than $11 \mathrm{eV}$ ) elements in the corona are a factor of 3-5 larger than in the photosphere (Meyer 1985a; Feldman 1992). Very low FIP (less than 6.1 eV) elements may have an even higher abundance ratio than the "normal" low-FIP elements (see, for example, Doschek, Feldman, \& Seely 1985; Phillips \& Feldman 1991; Feldman 1992).

The low-FIP to high-FIP enhancement factor seem to depend on the region of the corona under consideration. Widing \& Feldman (1992) found the relative abundance of $\mathrm{Mg}$ (low-FIP) to $\mathrm{Ne}$ (high-FIP) to Ne (high-FIP) in polar plumes to be as much as a factor of 10 greater than the photospheric ratio. The Meyer (1993) "coronal" abundances have a low-FIP to high-FIP abundance ratio approximately 4.5 greater than the photospheric ratio. McKenzie \& Feldman (1992) and Strong, Lemen, \& Linford (1991) showed that the ratio of abundances of low-FIP to high-FIP elements in active regions can vary from photospheric values to Meyer "coronal" abundance values. Doschek et al. (1985) and Phillips \& Feldman (1991) have reported that in plumes and impulsive flares, elements with very low FIP show a factor of 2 abundance increase over other low-FIP elements.

The question of whether low-FIP elements are enhanced or high-FIP elements are depleted relative to hydrogen is

\footnotetext{
${ }^{1}$ NASA/Marshall Space Flight Center, ES82, Huntsville, AL 35812.
}

still open to debate. Since hydrogen is by far the most abundant element, it should be taken as our reference. At coronal temperatures hydrogen is completely ionized, and direct measurements are difficult. The hydrogen first, and only, ionization potential is $13.6 \mathrm{eV}$, which would make it a highFIP element by our definition. Early work by Veck \& Parkinson (1981) suggested that hydrogen acted as a low-FIP element, so that the high-FIP elements are depleted relative to hydrogen. More recent work has suggested that hydrogen probably acts as a high-FIP element, so that the low-FIP elements are enhanced (Meyer 1993; Stirling, Doschek, \& Feldman 1993), or that there is a combination of low-FIP enhancement and high-FIP depletion (Fludra \& Schmelz 1995).

Feldman (1992) proposed a two-step enrichment process in which the very low FIP elements are enriched in the first stage. The second stage then involves enriching all the low-FIP elements by an equal factor. This model would require all elements that are low-FIP (7.5-10 eV FIP) to have the same, relative to other low-FIP elements, both in the corona and in the photosphere.

It is important to understand the abundance variability in the corona for a variety of reasons. The relative elemental abundance of different coronal structures could be used as a diagnostic of the coronal plasma source site for the structure because at coronal temperatures elements are multiply ionized and thus the elements' FIP has little effect. The low-FIP enhancement probably occurs in plasma with temperatures around $10^{4} \mathrm{~K}$ in which the low-FIP elements are ionized and the high-FIP elements are neutral. Hence the elemental abundance enhancement occurs in the chromosphere or photosphere.

When spectroscopic diagnostics use spectral lines from different elements, a relative elemental abundance is assumed. These diagnostics compare the relative intensity of two different spectral lines to determine the electron density and temperature. Since the coronal elemental abundance can vary by an order of magnitude in the extreme cases, the result of the coronal diagnostic can be in error. Normally, the effect of relative elemental abundances is less 
for broad spectral band instruments such as Yohkoh, but the effect should still be taken into account. When determining differential emission measures, one of the variables is the relative elemental abundance.

To determine heating requirements of the solar corona, the radiation loss must be estimated. To estimate the total coronal emission from a portion of the corona, the electron density and the relative elemental abundance must be known. According to Cook et al. (1989), for temperatures between $3 \times 10^{5}$ and $3 \times 10^{6} \mathrm{~K}$, iron dominates the emission. Hence the iron to hydrogen abundance must be known in order to make accurate estimates of the total radiative flux.

\subsection{New Results}

The data other researchers have used to determine relative elemental abundances have been primarily either from solar wind particle experiments or from spectroscopy of bright compact features. The Solar EUV Rocket Telescope and Spectrograph (SERTS) instrument allows the determination of relative elemental abundance of the background quiet Sun. The observations analyzed in this paper were of a region off the solar limb above a quiet-Sun region. The relative elemental abundance of $\mathrm{Si}, \mathrm{Al}$, and $\mathrm{Cr}$ to $\mathrm{Fe}$ was determined. All are low-FIP elements except $\mathrm{Al}$, which is a very low FIP element $($ FIP $=5.55 \mathrm{eV})$.

We find that the relative elemental abundances of Si to Fe determined from SERTS 1989 observations agree with either photospheric abundances (Allen 1973) or "coronal" abundances (Meyer 1985b; Stone 1989; Meyer 1992, private communication). The relative elemental abundance determined from the SERTS 1991 observations did not agree with either photospheric abundances (Allen 1973) or "coronal" abundances (Meyer 1985b; Stone 1989; Meyer 1992, private communication). The abundance of the very low FIP element, Al, was enhanced compared to the abundances of the low-FIP elements, $\mathrm{Cr}, \mathrm{Fe}$, and $\mathrm{Si}$. This result indicates that the plasma source region for the quiet-solar corona may have a two-step enrichment process, as Feldman (1992) proposed.

\section{THEORY}

\subsection{The Atomic Physics of Coronal Emission Lines}

A variety of solar coronal properties, such as electron density, electron temperature, and relative elemental abundance, can be determined spectroscopically with knowledge of the emission mechanism of the spectral line. The spectral lines are primarily produced by electron collisional excitation, for which the intensity depends on the electron density squared.

The emission rate for electron collisional excitation per unit volume, $\epsilon$, is

$$
\epsilon_{X, Z, i, j}=Q_{\mathrm{X}, Z, i, j}\left(T_{e}, N_{e}\right) \frac{N_{\mathrm{X}}}{N_{\mathrm{H}}} \frac{N_{\mathrm{H}}}{N_{e}} N_{e}^{2},
$$

where $N_{\mathrm{H}} / N_{e}$, the ratio of hydrogen to electrons in the corona, is 0.83 (Feldman 1992), $N_{\mathrm{X}} / N_{\mathrm{H}}$ is the elemental abundance of element $\mathrm{X}$, and $Q_{\mathrm{X}, Z, i, j}\left(T_{e}, N_{e}\right)$ is called the contribution function and is determined theoretically, with $\mathrm{X}$ the element, $Z$ the ionization stage, $i$ the upper excitation level, and $j$ the lower excitation level. The contribution function is strongly dependent on the electron temperature, $T_{e}$, because of the ionization balance and normally is weakly dependent on the electron density, $N_{e}$. Values for the contribution function have been calculated by Monsignori-Fossi \& Landini (1995), Brickhouse, Raymond, \& Smith (1995), and Mewe, Gronenschild, \& van den Oord (1985), using the ionization balance calculations of either Arnaud \& Raymond (1992) or Arnaud \& Rothenflug (1985). Normally some set of elemental abundances is assumed in such calculations.

The values of $N_{e}, T_{e}$, and the relative elemental abundance can be determined by comparing the ratio of emissivities of different spectral lines. If we take the ratio of the emissivities of two spectral lines from the same element $X$ and ionization stage $Z$, we get

$$
\frac{\epsilon_{\mathrm{X}, Z, i, j}}{\epsilon_{\mathrm{X}, Z, k, l}}=\frac{Q_{\mathrm{X}, Z, i, j}\left(T_{e}, N_{e}\right)}{Q_{\mathrm{X}, Z, k, t}\left(T_{e}, N_{e}\right)} .
$$

Since both spectral lines are from the same ionization stage of the same element, they have almost the same temperature dependence but, in some cases, have different density dependencies; for such pairs the line-ratio density can be determined as shown in Figure 1.

Next, using spectral lines from different ionization stages of the same element, $T_{e}$, can be determined since $N_{e}$ is known. Uncertainty in the calculations of the intensity ratios that is due to the theoretical ionization balance can be as large as $50 \%$ times the square root of the difference in ionization stages. For example, the uncertainty in the intensity ratio of Fe XVI to Fe XIV could be $\sqrt{2} \times 50 \%$, (Arnaud 1994; Raymond 1994; Monsignori-Fossi 1994, all private communications). The line ratio can change by 2 orders of magnitude over a factor of 2 change in the temperature (Fig. 2), so that reasonable line-ratio temperatures can be determined. The spectral lines used in the ratio should be relatively density-insensitive. Such lines are normally the strongest spectral lines from the ionization stage. Once $N_{e}$ and $T_{e}$ are known, the relative elemental abundances can be

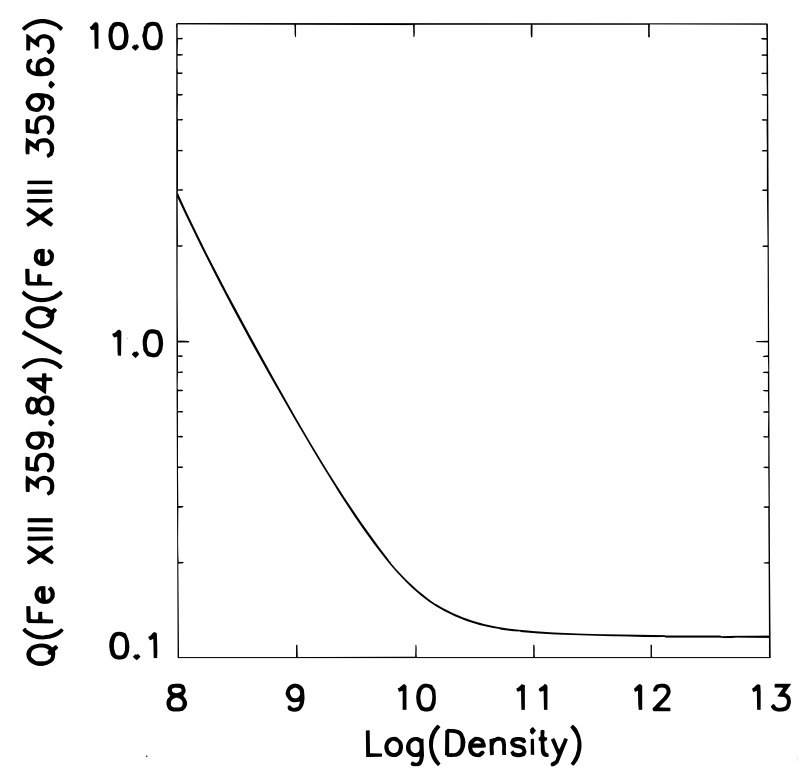

FIG. 1.-Theoretical ratio of intensities of Fe XIII $359.63 \AA$ and $359.84 \AA$ at $\log T_{e}=6.3$ (Monsignori-Fossi \& Landini 1995 emissivities). The ratio changes because as the density increases, the chance that the upper level of the Fe XIII $359.84 \AA$ transition is depopulated by collision instead of radiation increases. 


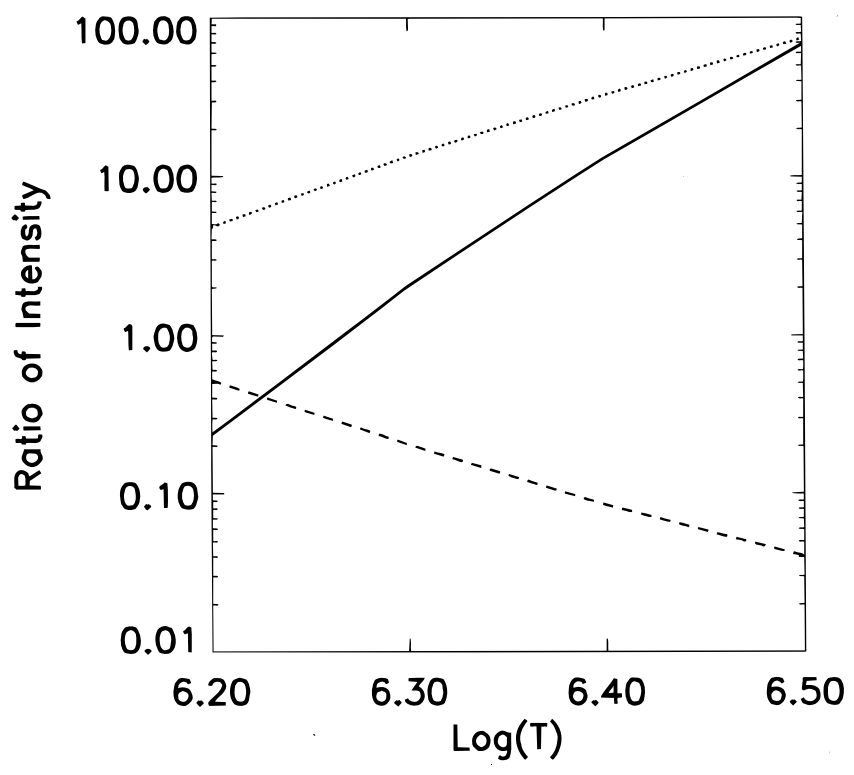

FIG. 2.-Theoretical line-ratio temperature assuming the Arnaud \& Rothenflug (1985) ionization balances for Fe XVI $361 \AA \AA / \mathrm{Fe}$ XIV $334 \AA$ (solid line), $\mathrm{Fe}$ XV $284 \AA / \mathrm{Fe}$ XIV $334 \AA$ (dotted line), and $\mathrm{Fe}$ XIII $359.63 \AA / \mathrm{Fe}$ XIV $334 \AA$ (dashed line).

determined by

$$
\frac{N_{\mathrm{X}_{1}}}{N_{\mathrm{X}_{2}}}=\frac{\epsilon_{\mathrm{X}_{1}, Z_{1}, i, j}}{\epsilon_{\mathrm{X}_{2}, Z_{2}, k, l}} \frac{Q_{\mathrm{X}_{2}, Z_{2}, k, l}\left(T_{e}, N_{e}\right)}{Q_{\mathrm{X} 1, Z_{1}, i, j}\left(T_{e}, N_{e}\right)} .
$$

\subsection{The Line-of-Sight Intensity}

The SERTS instrument, described in Neupert et al.(1992) and Thomas et al. (1991), was used to observe the corona off the solar limb as shown in Figure 3. The EUV (extremeultraviolet) emission lines observed were optically thin in the corona. The total intensity observed by the SERTS instrument is the integral over all the emissions directed along the line of sight toward the observer. The region closest to the Sun along a given line of sight will normally dominate because the spectral-line intensity is dependent on the square of the density and the density falls off exponentially with height.

The observed spectral-line intensity is the emission per unit volume integrated along the line of sight,

$$
I_{\mathrm{X}, Z, i, j}=\frac{1}{4 \pi} \int_{-\infty}^{\infty} \epsilon_{\mathrm{X}, Z, i, j} d x \quad \text { ergs } \mathrm{cm}^{-2} \mathrm{~s}^{-1} \mathrm{sr}^{-1},
$$

where $x$ is the path length along the line of sight and $x=0$ is the point along the line of sight closest to the Sun (Fig. 3). Substituting for $\epsilon_{\mathrm{X}, Z, i, j}$ with equation (1) and assuming that the elemental abundance along the line of sight is constant, we have

$$
\begin{aligned}
I_{\mathrm{X} . Z, i, j}= & \frac{\overline{Q_{\mathrm{X}, Z, i, j}\left(T_{e}, N_{e}\right)}}{4 \pi} \frac{N_{\mathrm{X}}}{N_{e}} \\
& \times \int_{-\infty}^{\infty} N_{e}^{2} d x \text { ergs cm } \mathrm{cm}^{-2} \mathrm{~s}^{-1} \mathrm{sr}^{-1} .
\end{aligned}
$$

The density-weighted average contribution function is defined as

$$
\overline{Q_{\mathrm{X}, Z, i, j}\left(T_{e}, N_{e}\right)} \equiv \frac{\int_{-\infty}^{\infty} Q_{\mathrm{X}, Z, i, j} N_{e}^{2} d x}{\int_{-\infty}^{\infty} N_{e}^{2} d x},
$$

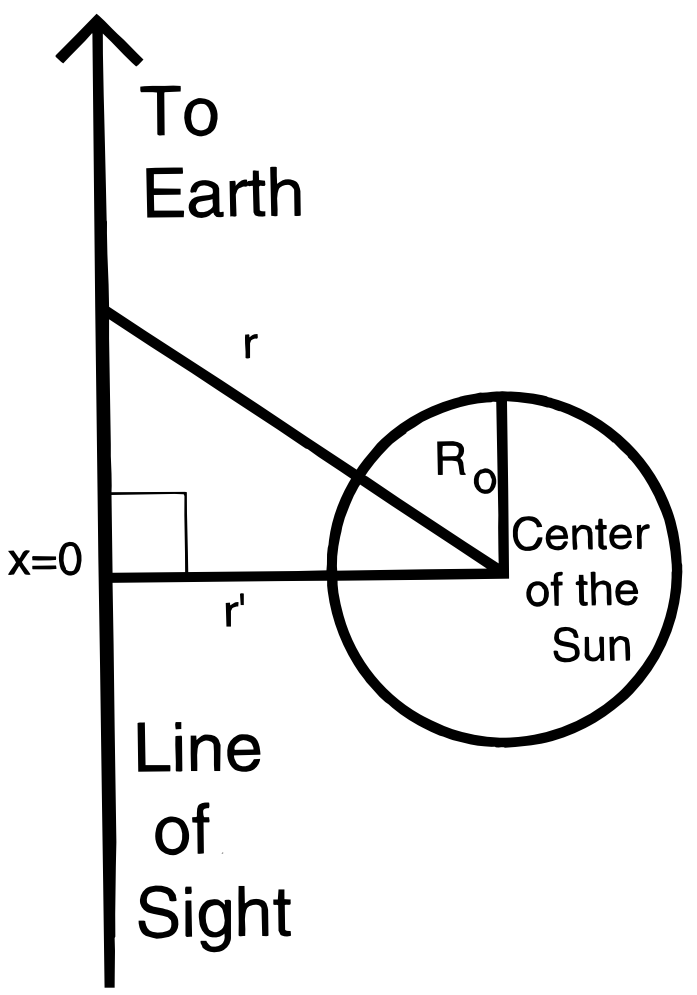

FIG. 3.- Line of sight to the Sun and other geometrical quantities

and we use the approximation

$$
\overline{Q_{\mathrm{X}, Z, i, j}\left(T_{e}, N_{e}\right)} \approx Q_{\mathrm{X}, Z, i, j}\left(\overline{T_{e}}, \overline{N_{e}}\right),
$$

where $\bar{T}_{e}$ is the average line-of-sight temperature and $\bar{N}_{e}$ is the average line-of-sight electron density.

The equation for the intensity along the line of sight is obtained by substituting equation (7) into equation (5), which yields

$$
I_{\mathrm{X}, Z, i, j} \approx \frac{Q_{\mathrm{X}, Z, i, j}\left(\overline{T_{e}}, \overline{N_{e}}\right)}{4 \pi} \frac{N_{\mathrm{X}}}{N_{e}} \eta \quad \operatorname{ergs~}^{\mathrm{cm}^{-2} \mathrm{~s}^{-1},}
$$

where $\eta$ is the column emission measure $\left(\eta=\int_{-\infty}^{\infty} N_{e}^{2} d x\right)$.

By taking ratios of the intensities of different spectral lines, the line-of-sight weighted average of $N_{e}$ and $T_{e}$ and the relative elemental abundance can be determined. These parameters do not depend on the absolute intensity but on the relative intensity of the spectral lines. If the absolute spectral line intensity is known, the quantity $N_{\mathrm{X}} / N_{e} \eta$ can be determined.

\section{OBSERVATIONS}

\subsection{Instrument}

The SERTS instrument obtains both images and spectra of solar coronal regions recorded on film. The film records images in emission lines from 235 to $450 \AA$ in first order and from 170 to $225 \AA$ in second order. The 1989 SERTS instrument was described in Neupert et al. (1992). The SERTS 1991 instrument modifications were described by Thomas et al. (1991). The pointing positions were determined by the method described by Brosius et al. (1993). The SERTS images were digitized and converted to exposure units as described by Thompson et al. (1993). Then instrumental efficiency and atmospheric extinction were taken into 
account. Finally, a transformation from relative to absolute solar irradiance was performed by normalizing the combined He II and Si XI $304 \AA$ line intensity to previously observed average quiet-Sun values, as described in Thomas $\&$ Neupert (1994). We estimate a factor of 2 uncertainty in the absolute calibration, which affects only the absolute values of the emission measure, not the relative values.

\subsection{Spectrograph Data Format}

The SERTS instrument obtains both slit spectra and spectroheliograms. The slit spectra observations only extend up to heights of $1.05 R_{\odot}$, but we have spectroheliograms from both the SERTS 1989 flight and the SERTS 1991 flight that contain observations to heights of $1.15 R_{\odot}$, which we have analyzed for this paper. First, we must explain the nature of the SERTS spectrograph and what our slit specta and spectroheliograms consist of.

A section of the spectrograph data from the 1991 SERTS flight is shown in Figure 4. Three different regions in the spectrograph data are readily apparent. A standard slit spectrum resides in the middle region, where spatial information is recorded only in the vertical direction and wavelength information is recorded only in the horizontal direction. The upper and lower regions are produced by the instrument lobes (Fig. 5), where the vertical axis is still purely spatial but the horizontal axis contains both spatial and wavelength information. We call such an image a spectroheliogram. Because of domination of the spectrum in the EUV by strong spectral lines and the nature of our entrance aperture (Fig. 5), we can often separate the spatial and wavelength dependences. This separation is easiest for bright spectral lines that have no other bright overlapping images.

\subsection{Determination of Spectral Line Intensity}

Next we will show how we determine the spectral line intensity. In Figure 4, edges of bright lobes are readily apparent. We can determine the intensity of the spectral line by determining the increase in brightness across the edge of the lobe. A possible intensity profile is shown in the left panel of Figure 5 to illustrate this method. Since the background is composed of both light scatter in the instrument and a large number of very weak spectral lines, the background is not constant. But we can determine the background from near the edge of the lobe and assume it to be equal to the background at the edge of the lobe. To ensure that this assumption is valid, we check that no strong or medium-strength spectral line has the opposite edge of its lobe ending between where we take the background and the edge of the lobe. Since we know the effective width of the lobe in angstroms, this check is easily made.

If the edge of the lobe were a step function, we could determine the background from a point directly adjacent to the edge of the lobe. In reality, though, we must determine the background from a point farther from the edge (Fig. 6). The larger the separation between the position at which we determine the background and that at which we measure the intensity of the lobe, the greater is the potential uncertainty in the determination of the spectral line intensity. Hence we limit ourselves to determining the intensity along the edge of the lobe, which gives us the intensity as a function of height.

\section{Fe XV 284A He II 303A}

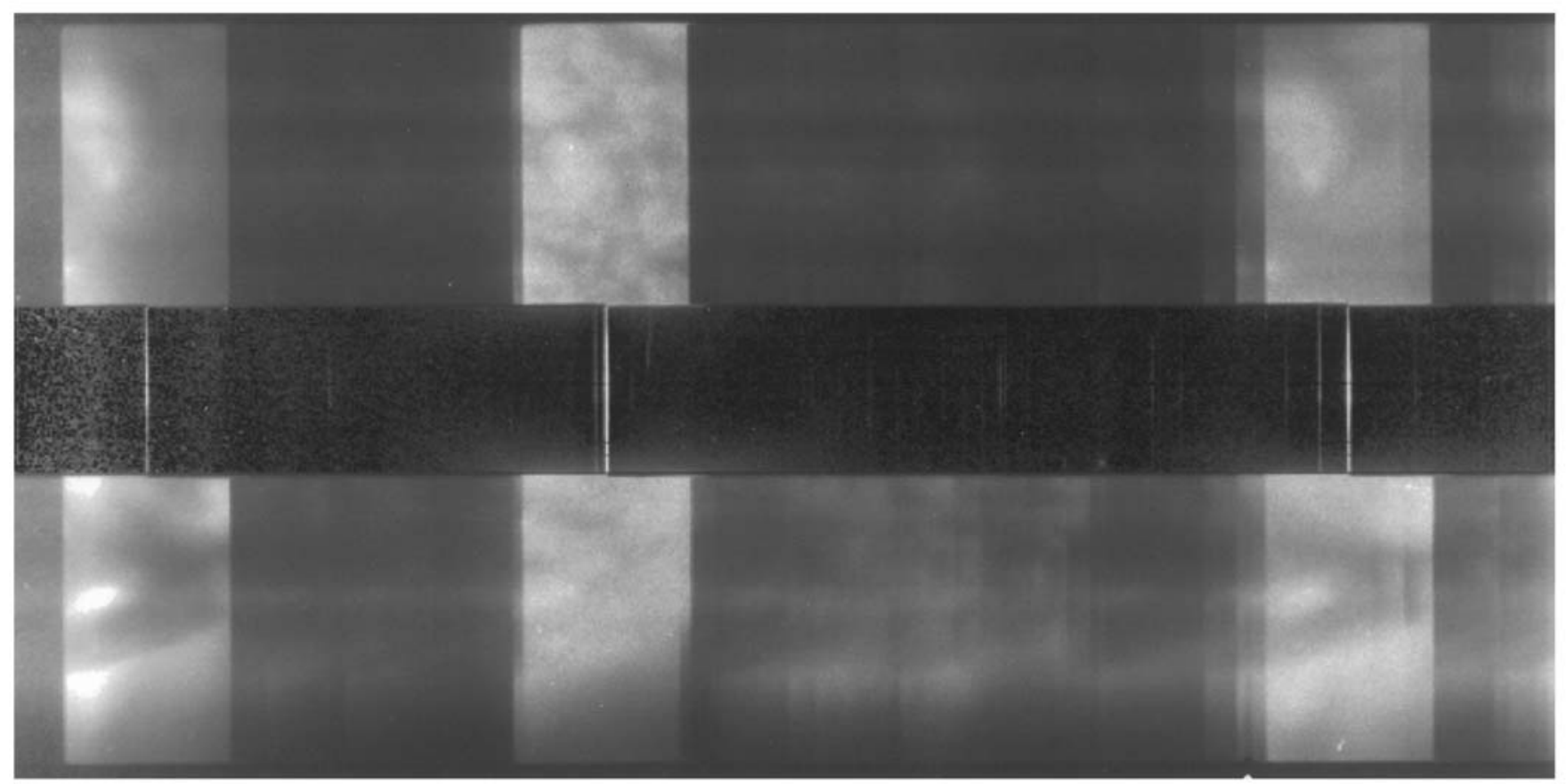

FIG. 4.-Part of a SERTS 1991 spectrum, with the intensity of the narrow-slit spectra enhanced (central region). Additional spectral lines exist but are too faint to see in the brightness scale shown. The upper and lower lobes permit us to image regions of the Sun in many different spectral lines as well as the corona above the solar limb (lower lobe). There is some overlapping of the wide lobes, but the brightest spectral lines, which are labeled, produce easily observed edges. By measuring the increase of intensity across the edge of the lobe we can determine the spectral line intensity (Fig. 5). 


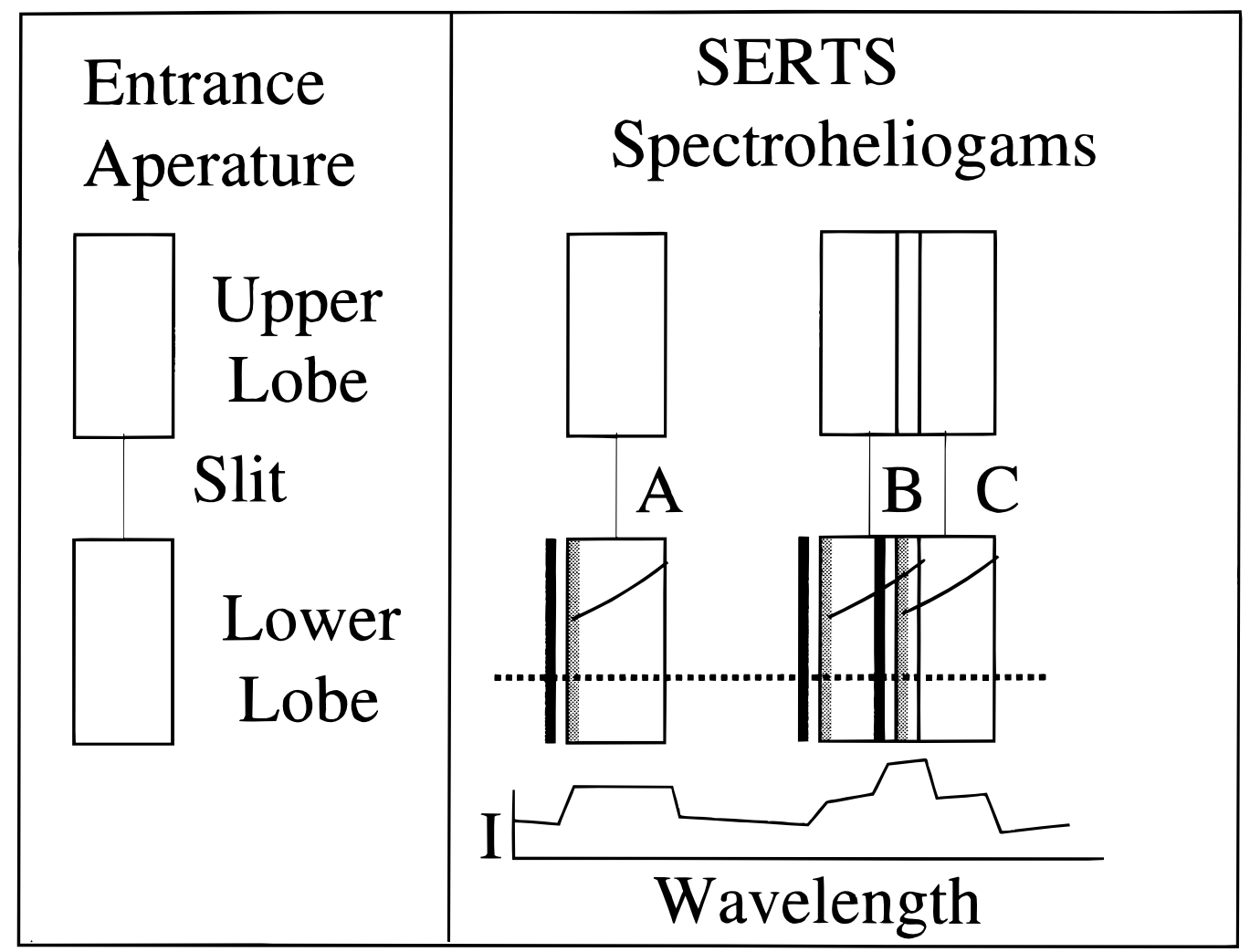

FIG. 5.-Left panel: schematic of the SERTS entrance aperture, which consists of an upper lobe, a lower lobe, and a slit. This entrance aperture is reproduced for each spectral line shown in Fig. 4, as shown schematically in the right panel. (The limb of the Sun is drawn in for each spectral line.) Spectral line A is an example of a well-isolated spectral line, while spectral lines B and C are not well isolated, so that their lobes overlap (since the wavelength difference between lines B and C is less than $6.2 \AA$ ). The intensity along the dotted line produces the intensity profile shown at the bottom of the right panel. Note that the edge of the lobe is not a step function and that the background intensity is never zero because of the presence of scattered light in the instrument and overlapping images from weak spectral lines. We can determine the intensity of the spectral line by measuring the increase of intensity across the edge of the lobe, as described in $\S 3.3$. We find the intensity by taking the difference of a background strip (dark gray) and the edge of the lobe (light gray) when the background strip is separated from the edge of the lobe far enough to minimize the scatter of emission from the edge of the lobe.

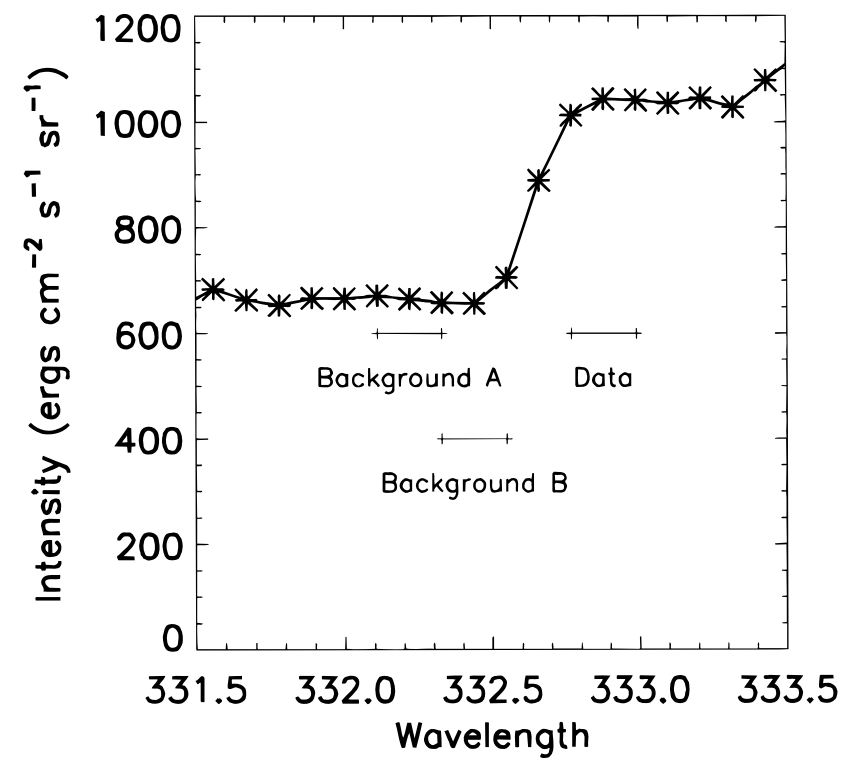

Fig. $6 a$

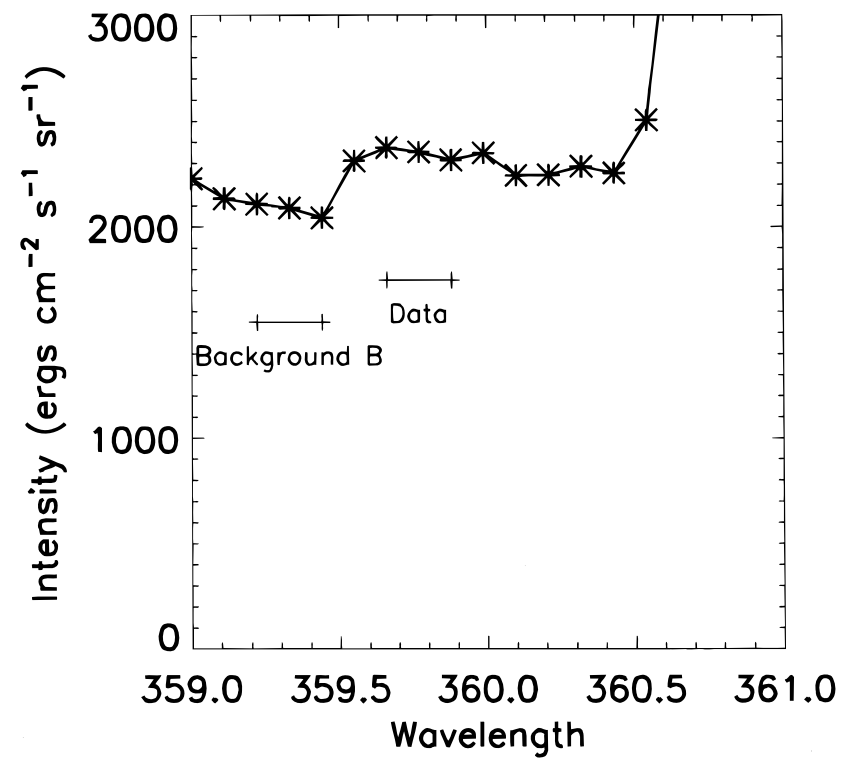

FIG. $6 b$

FIG. 6.-(a) Spectrum for a fraction of the lobe for the $\mathrm{Al} \times 332 \AA$ spectral line. (The wavelength scale in the abscissa is aligned with the southern edge of the lobe.) Because of scatter in the instrument, the background must be taken four to six $50 \times 50 \mu \mathrm{m}$ pixels from the edge of the lobe (background A). The $\mathrm{Al} \mathrm{x}$ intensity, which is the difference between the part of the spectra marked "data" and background A, is about $400 \mathrm{ergs} \mathrm{cm}^{-2} \mathrm{~s}^{-1} \mathrm{sr}^{-1}$. (b) For the Fe XIII $359 \AA$ line, the lobe from a nearby spectral line forces us to take the background two to four pixels from the edge of the lobe (background B). From analysis of different lines, we estimate that the background is increased by $5 \%$ of the spectral line intensity in $(b)$ over its value in $(a)$. The edge of the lobe from the Fe XVI $361 \AA$ line can be seen on the right at about $360.5 \AA$. 
The digitized data were merged into $150 \times 150 \mu \mathrm{m}^{2}$ pixels $\left(13^{\prime \prime} \times 13^{\prime \prime}\right)$ that are used for the analysis in this paper. For each spectral line, we determine the intensity of a strip along the edge of the lobe. This intensity is the sum of the spectral-line intensity and the background. The background is determined from a similar strip of pixels three $13^{\prime \prime} \times 13^{\prime \prime}$ pixels from the edge of the lobe (Fig. 6).

The relative uncertainty in the spectral-line intensity determined by this method is around $15 \%$ because of uncertainties in intensity calibration, background subtraction, and the determination of the position of the edge of the lobe. Except for weak lines, the variation due to noise in the intensity of the spectral lines with radius is normally less than the systematic uncertainties. The $15 \%$ uncertainty is the systematic uncertainty in the overall intensity of the spectral line, rather than that of an individual spectral pixel on the line.

\subsection{Line Selection and Determination of Spectral-Line Intensity}

In order for analysis of the SERTS off-the-limb data to be accurate, a spectral line must meet the following conditions:

1. The line should be strong compared to the background, in order to minimize uncertainties in the line intensity.

2. Adjacent lines must be resolved spectrally. When two ions emit at nearly the same wavelength, their emission lines blend and only one spectral line is observed. This observed line is a combination of the two blended lines, which makes analysis of the combined radiation difficult because of large uncertainties in the individual line intensities.

3. The emission mechanism must be well understood. If the line emission mechanism is not well understood, or if the emission can be produced by several processes of similar strength (e.g., electron collision, resonance scattering, and recombination), the analysis of the line will be uncertain. Such spectral lines were not included in this study.

4. Any overlapping images should be weak, since they create additional uncertainties in the spectral-line intensity.

\subsubsection{The SERTS 1991 Flight}

The southern edge of the off-the-limb portion of the lobes on the SERTS 1991 flight was found to have six spectral lines that satisfied the selection criteria. These lines were the Al x $332 \AA$, Cr XIII $328 \AA$, Si XI $303 \AA$, Fe XIII $359 \AA$, Fe XIV $334 \AA$, and Fe XVI $361 \AA$ lines. This data set will be referred to as the SERTS 1991 southern edge. The intensity as a function of height for the different spectral lines is shown in Figure 7.

The Fe XIII 359 Å line had the additional complication of a nearby lobe overlapping the region in which the background normally would be measured. Since the Fe XIII line was useful for this analysis for confirming the line-ratio temperature measurements, special treatment was given to this line. The background was determined using $13^{\prime \prime} \times 13^{\prime \prime}$ pixels that were only two pixels away from the lobe edge, instead of the normal three pixels away. Since this background is not the true background because it is also affected by scattered light from the Fe XIII line, the intensity of the $\mathrm{Fe}$ XIII line is clearly underestimated. To determine the correction factor, a study of other spectral lines was performed by taking the background closer to the lobe edge. As was

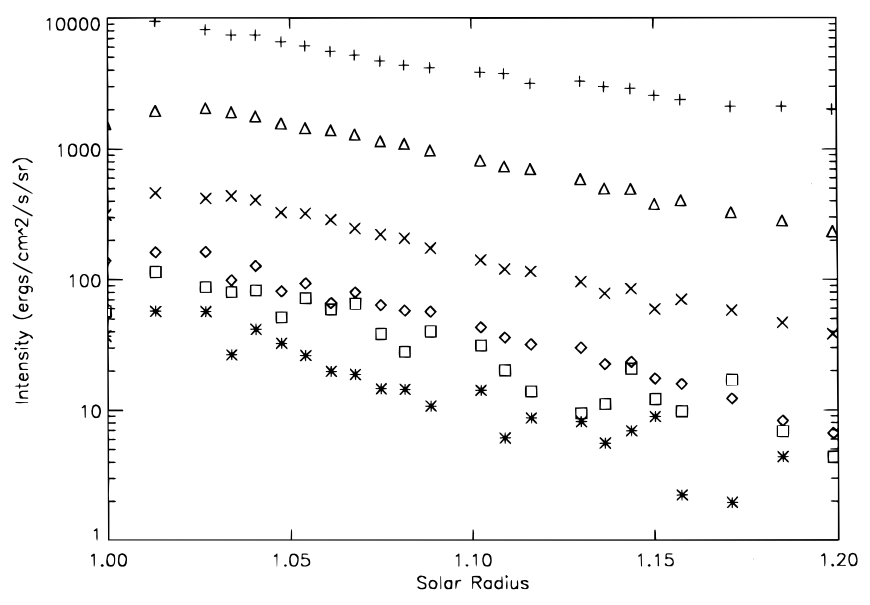

FIG. $7 a$

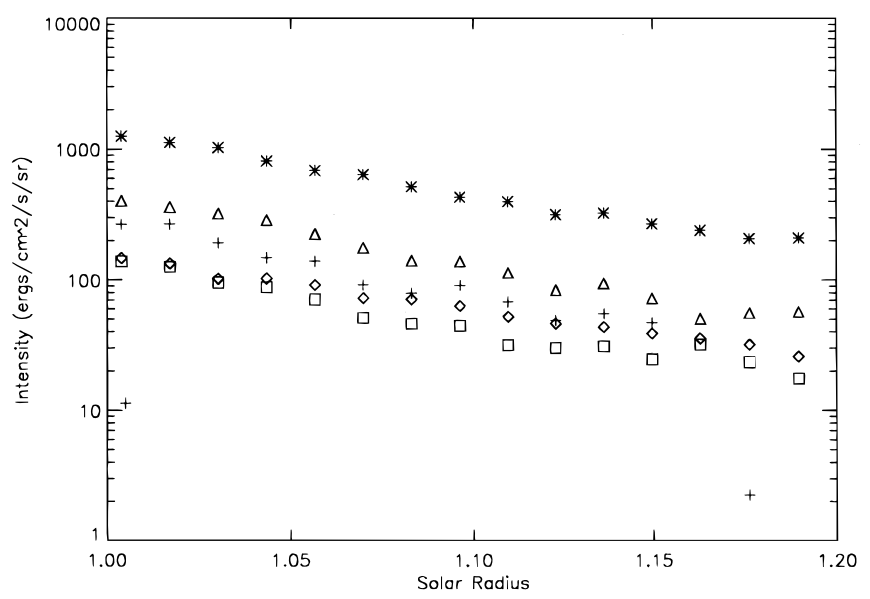

FIG. $7 b$

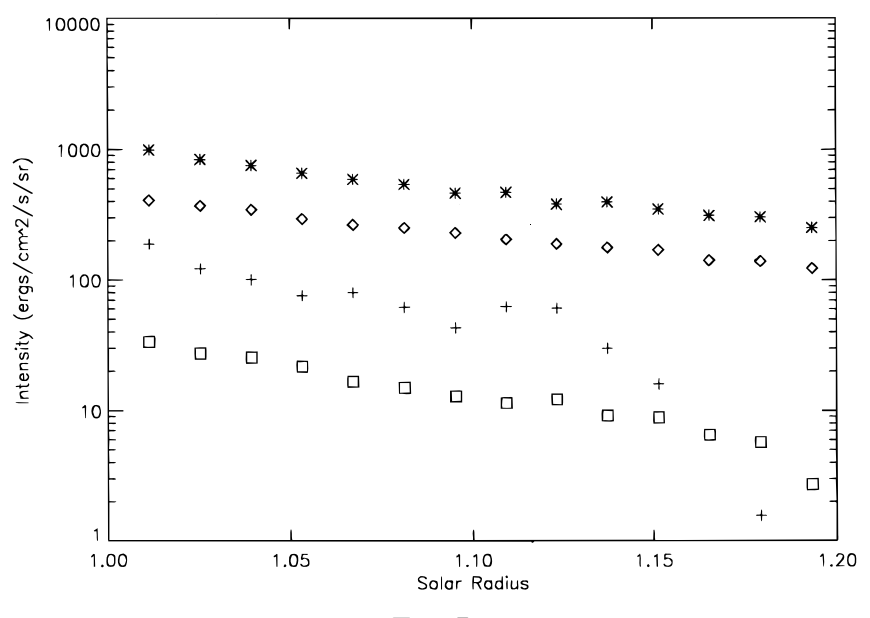

FIG. $7 c$

FIG. 7.- - Log intensity vs. radius for (a) the SERTS 1991 southern edge, (b) the SERTS 1989 southern edge, and (c) the SERTS 1989 northern edge. In (a), the spectral-line intensities shown are for the Fe XVI $361 \AA(+)$, Fe XIV $334 \AA(\times)$, Fe XIII $359.63 \AA(\square)$, Cr XIII $328 \AA$ (*), Si XI $303 \AA(\triangle)$, and $\mathrm{Al} \times 332 \AA(\diamond)$ lines. The spectral lines with the weakest intensities have the largest signal-to-noise ratio. In $(b)$, the spectral-line intensities shown are for the Fe XVI $361 \AA$ (dia.), Fe Xv $284 \AA$ (*), Fe XIV $334 \AA \AA$ ( $\square$ ), Fe XIV $274 \AA(+)$, and Si XI $303 \AA(\triangle)$ lines. In $(c)$, the spectral-line intensities shown are for the Fe XVI $361 \AA(\triangle), F e$ Xv $284 \AA(*), F e$ Xv $417 \AA(\square)$, and Fe XIV $274 \AA(+)$ lines. Beyond $1.15 R_{\odot}$ the Fe XIV $274 \AA$ intensity is unreliable in $(b)$ and $(c)$. 
TABLE 1

SPECTRAL LINE INFORMATION

\begin{tabular}{|c|c|c|c|c|}
\hline Ion & $\begin{array}{l}\text { Wavelength } \\
\text { (Å) }\end{array}$ & $\begin{array}{l}\text { Oscillator } \\
\text { Strength }^{\mathrm{a}}\end{array}$ & Transition $^{\mathrm{b}}$ & $\begin{array}{c}\text { Ionization Balance } \\
\text { References }\end{array}$ \\
\hline $\mathrm{Al} \mathrm{x} \ldots \ldots \ldots$ & 332 & 0.287 & $2 s^{2}{ }^{1} S_{0}-2 s 2 p{ }^{1} P_{1}^{o}$ & 1 \\
\hline Cr XIII....... & 328 & 0.902 & $3 s^{2}{ }^{1} S_{0}-3 s 3 p^{1} P_{1}^{o}$ & 2 \\
\hline Si XI ......... & 303 & 0.264 & $2 s^{2}{ }^{1} S_{0}-2 s 2 p^{1} P_{1}^{o}$ & 1 \\
\hline Fe XIII....... & 359.63 & 0.048 & $3 s^{2} 3 p^{23} P_{1}-3 s 3 p^{3} D^{o}$ & 1,3 \\
\hline Fe XIV ....... & 274 & 0.200 & $3 s^{2} 3 p^{2} P_{1 / 2}^{o}-3 s 3 p^{2}{ }^{2} S_{1 / 2}$ & 1,3 \\
\hline Fe XIV ......... & 334 & 0.077 & $3 s^{2} 3 p^{2} P_{1 / 2}^{o}-3 s 3 p^{2}{ }^{2} D_{3 / 2}^{1 / 2}$ & 1,3 \\
\hline $\mathrm{Fe} \mathrm{xV} \ldots \ldots$ & 284 & 0.827 & $3 s^{21} S_{0}-3 s 3 p^{1} P_{1}^{o}$ & 1,3 \\
\hline $\mathrm{Fe} \mathrm{xv} \ldots \ldots$ & 417 & 0.032 & $3 s^{2}{ }^{1} S_{0}-3 s 3 p^{3} P_{1}^{o}$ & 1,3 \\
\hline Fe XVI...... & 335 & 0.270 & $3 s^{2} S_{1 / 2}-3 p^{2} P_{3 / 2}^{o}$ & 1,3 \\
\hline Fe XVI....... & 360 & 0.125 & $3 s^{2} S_{1 / 2}-3 p^{2} P_{1 / 2}^{o}$ & 1,3 \\
\hline
\end{tabular}

${ }^{\text {a }}$ From Fuhr et al. 1988, Martin et al. 1988, and Wiese et al. 1969.

${ }^{\text {b }}$ From Kelly 1987.

REFERENCES.-(1) Arnaud \& Rothenflug 1985. (2) Monsignori-Fossi \& Landini 1995. (3) Arnaud \& Raymond 1992.

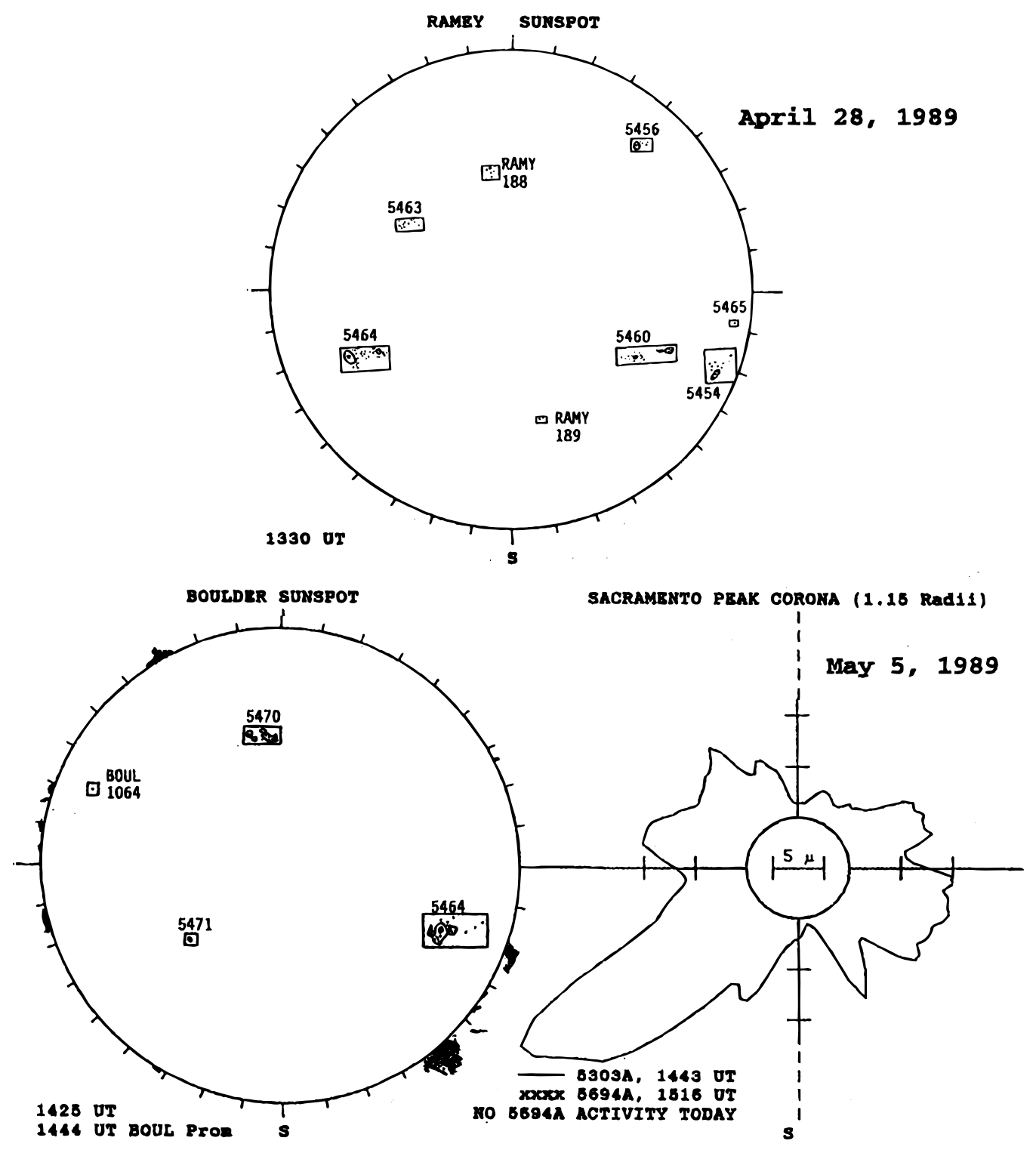

FIG. 8.-Top: Active regions on the Sun one-quarter of a solar rotation before the SERTS 1989 flight. Bottom left: Location of the active regions on the day of the flight. Bottom right: Coronagram from the day of the flight. The location of the edges of the lobe was on the eastern limb. Source: Sol-Geophys. Data (1989a, 1989b). 


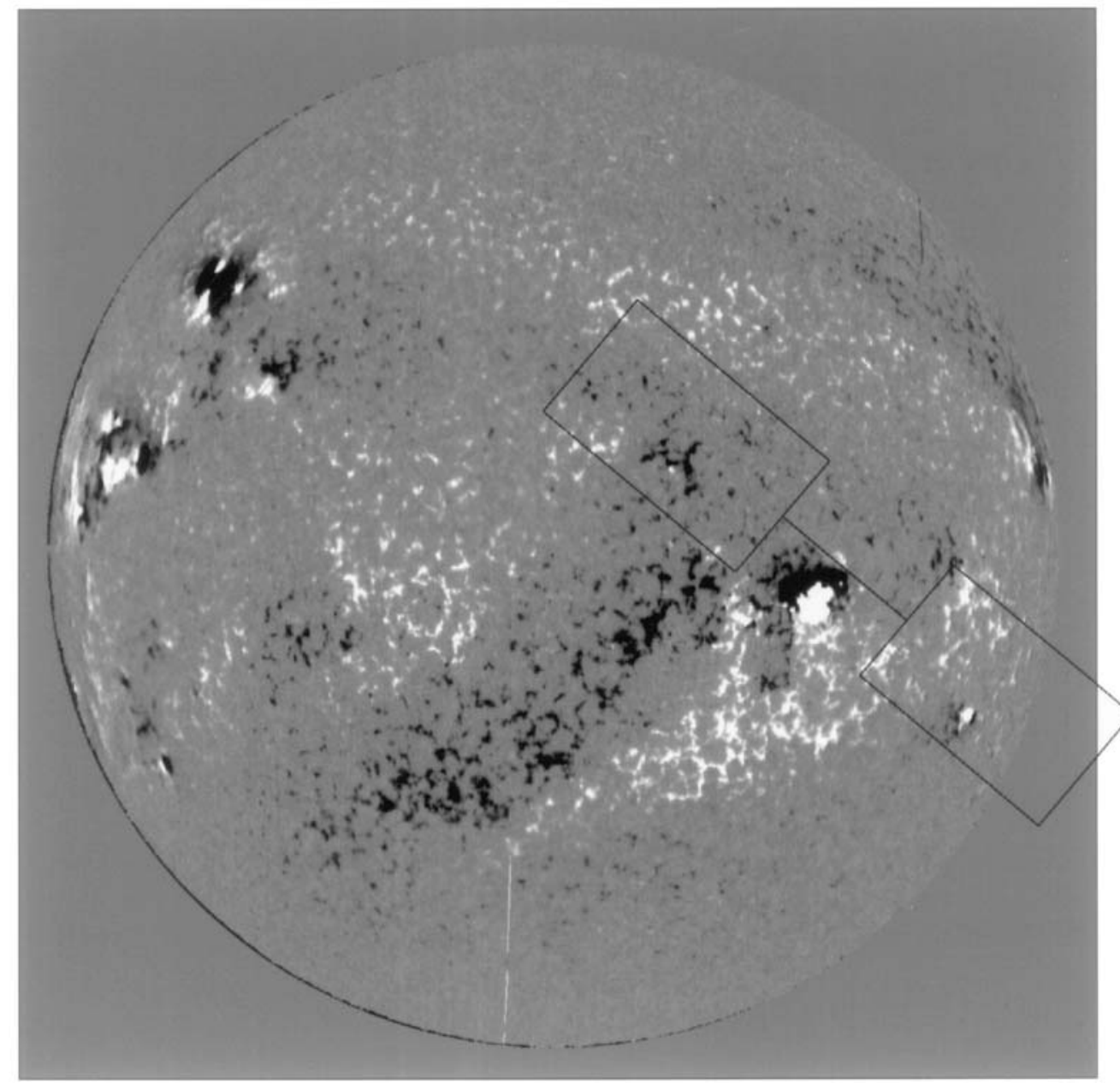

FIG. 9.-SERTS 1991 pointing position superimposed on a solar magnetogram (Brosius et al. 1993). The second pointing position was shifted along the main axis of the lobes to extend further off the solar limb. The white and black regions on the magnetogram indicate the sign of the line-of-sight component of the photospheric magnetic field.

done for Fe XIII, the correction factor was determined to be about 1.1 (Figs. $6 a, 6 b$ ). The Fe XIII line was then assigned an uncertainty of $25 \%$, instead of the normal $15 \%$, because of the extra uncertainty and the other weak overlapping lobes.

In the SERTS 1991 data, the overlapping portions of the lobe's edge from the two different pointing positions were merged. Pointing position 1 covered the corona in the range $1.047 \leq r \leq 1.525$. Pointing position 2 extended this region to cover heights of $1.00 \leq r \leq 1.15$. The combination of these data permits us to extend the analysis of the solar corona to greater heights than either one of the pointing positions alone would have allowed. Because many lines had weak intensities, the analysis covered between $1.0 \leq r \leq 1.2$. Since the spectral-line intensity did not align smoothly because of calibration uncertainties, a boxcar smoothing of five $13 \times 13 \operatorname{arcsec}^{2}$ pixels was used in the derived radial profiles.

\subsubsection{The SERTS 1989 Flight}

The southern edge of the off-the-limb portion of the SERTS 1989 lobes was found to have five spectral lines that satisfied the selection criteria: the Si XI $303 \AA$, Fe XIV $274 \AA$, $\mathrm{Fe}$ XIV $334 \AA$, Fe XV $284 \AA$, and Fe XVI $361 \AA$ lines. The northern edge had four spectral lines, namely the Fe XIV 274 $\AA ̊ ., F e$ XV $284 \AA, F e$ Xv $417 \AA$, and Fe xvI $335 \AA$ lines. The reasons for the changes in lines between the two flights were the wider wavelength coverage in the SERTS 1989 data, and the increased efficiency and intensity of emission in the SERTS 1991 data. A list of the spectral lines used, the ion that produces each line, the wavelength, oscillator strength, and transition array, and the ionization balance calculations are given in Table 1 . The intensity as a function of height for the different spectral lines is shown in Figures $7 b$ and $7 c$.

\subsection{Observations}

Next we will describe the conditions that existed off the solar limb during the two flights for the regions observed. These conditions were deduced from sunspot and coronagraph observations from Solar-Geophysical Data (1989a, 1989b, 1991) and from SERTS lobe images from different spectral lines.

\subsubsection{The SERTS 1989 Flight}

The SERTS 1989 flight had only one of the two pointing positions partially off the limb, as is discussed in more detail, including a review of the observations and pointing position, in Neupert et al. (1992). Since only one exposure in this pointing position was made, only this exposure was considered. Both edges of the lobe off the solar limb were used for this study. The southern edge will be referred to as the SERTS 1989 southern edge, the northern edge as the SERTS 1989 northern edge. The sunspot activity is shown in Figure 8. The bottom left panel of Figure 8 shows the 

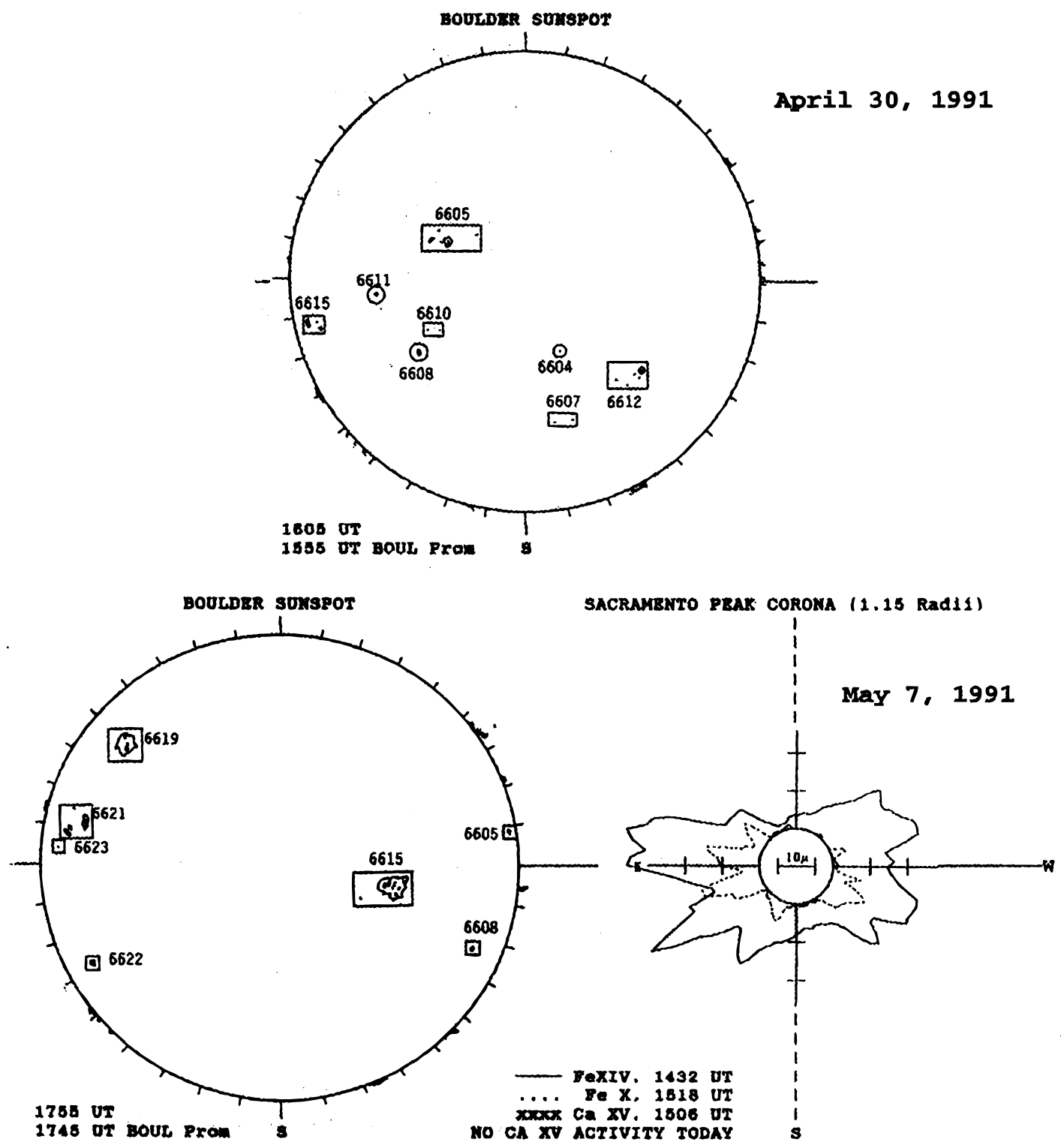

FIG. 10.-Same as Fig. 8, but for the SERTS 1991 flight. The location of the edges of the lobe can be seen in Fig. 9. Source: Sol.-Geophys. Data (1991).

sunspot map for the day of the flight. The upper panel shows the sunspot map from a quarter of a solar rotation earlier in order to show the general nature of the solar activity behind the limb on the day of the flight. The sunspot maps show that Active Regions 5464 and 5460 lay near the line of sight. The lower right panel shows the Fe XIV Sacramento Peak coronagram; no Fe x coronagram was made for the flight date. The figure indicates that the two observed regions analyzed here should be classified as quiet solar corona.

\subsubsection{The SERTS 1991 Flight}

The SERTS 1991 flight had two different pointing positions, each with four exposures of different durations. In the first pointing position, the lower (outer) lobe was completely off the solar limb, with a part of the narrow slit off the disk. In the second pointing position, only a fraction of the outer lobe was off the disk. The longest exposure for each pointing position was used for our analysis, or else, when some lines were overexposed, the second-longest exposure was used.
Only the southern edge of the lobe was used for this study because of the lack of a reasonable number of strong iron spectral lines on the northern edge of the lobe. This edge will be called the SERTS 1991 southern edge (see Fig. 9). While there were no active regions at the solar limb, there were active regions in the foreground and others had rotated around the solar limb, as can be deduced from the top panel of Figure 10, which shows the activity onequarter of a solar rotation earlier. The Fe XIV coronagram of SERTS 1991 shows larger emission than that from the SERTS 1989 flight. A hot loop is seen near the southern edge of the lobe, as well as a number of foreground and background active regions. The coronagrams taken for the day of the flight show both Fe $\mathrm{x}$ and Fe XIV emissions off the solar limb. These observations are classified as coming from a more active quiet-Sun region, since more emission from the active regions is seen along the line of sight.

\section{ANALYSIS}

To determine the relative elemental abundance from the radial dependence of the spectral line intensity, the follow- 
ing steps are required: (1) determine limits on $\overline{N_{e}}$, (2) determine the radial dependence of $\overline{T_{e}}$, and (3) determine the column emission measure $\eta$. The relative elemental abundance can then be determined.

\subsection{Line-Ratio Electron Density}

For the SERTS 1991 southern edge, the nondetection of the Fe XIV 353 Å line along with an observable Fe XIV $334 \AA$ line indicates that the density must be less than $10^{10} \mathrm{~cm}^{-3}$. Similarly, lower density limit of greater than $10^{9} \mathrm{~cm}^{-3}$ can be determined since the Fe XIII $359.63 \AA$ was observed and the Fe XIII $359.84 \AA$ line was not.

An upper limit on the electron density for the SERTS 1989 flight was determined by observing that the Fe XIV 264 $\AA$ and the Fe XIV $274 \AA$ intensities were similar, which puts a strong upper limit of $10^{10} \mathrm{~cm}^{-3}$ on the density. The density is probably much less than $10^{10} \mathrm{~cm}^{-3}$, as this limit is sensitive to the large background noise near the $264 \AA$ Aline.

\subsection{Line-Ratio Temperature}

The line-ratio temperature was determined using the method described in $\S \S 2.1$ and 2.2 for spectral lines from different ionization stages of iron. Densities consistent with the limits on the electron density determined in $\S 4.1$ were used. The measured line-ratio temperatures for the SERTS 1989 southern edge are shown in Figure 11 and were determined using Arnaud \& Rothenflug's (1985) ionization balance and Monsignori-Fossi \& Landini's (1995) emissivities.

The systematic temperature uncertainties due to instrumental and theoretical uncertainties are shown in Figure 11 as vertical error bars in the inset panel; they affect the overall normalization of the derived line-ratio temperature curves but do not give the relative uncertainties of each data point. This systematic uncertainty will dominate both the emission measure and the relative elemental abundance.

Different sets of spectral lines give different line-ratio temperatures, but the differences are less than the systematic uncertainties. A pattern is apparent for line-ratio temperatures from any one set of spectral lines. The line-ratio temperature increases roughly linearly as a function of radius. A fit of the form

$$
T=T_{0}+a(r-1)
$$

was applied to the line-ratio temperature for each set of iron spectral lines. In Figure 11, the fits to the line-ratio temperatures are shown as solid lines. The fit to the Fe XIV-XVI lines was selected as the most accurate fit because it had the lowest uncertainties. This fit will be used as the radial

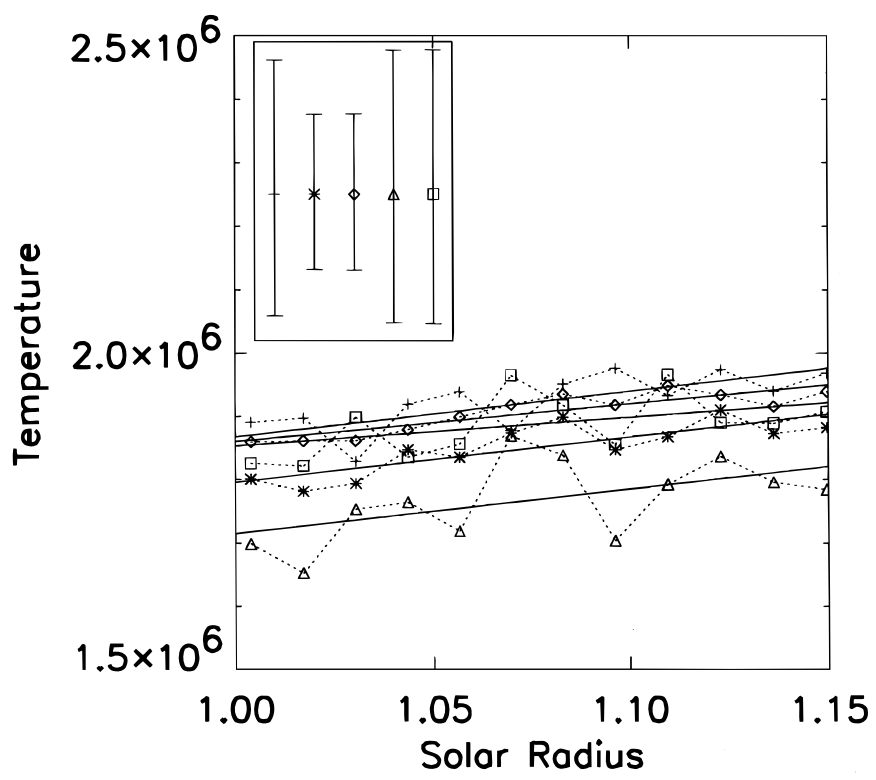

FIG. 11.-Line-ratio temperature vs. radius for the SERTS 1989 southern edge. The line-ratio temperature is calculated using the Arnaud \& Rothenflug (1985) ionization balance. The following measured lineratio temperatures are plotted: Fe XV $284 \AA$ \&/Fe XVI $360 \AA$ (+), Fe XIV $274 \AA$ 的 XVI $360 \AA$ (*), Fe xIV $334 \AA / \mathrm{Fe} 360 \AA(\diamond), \mathrm{Fe}$ xIV $274 \AA / \mathrm{Fe}$ xV $284 \AA(\triangle)$, and Fe xIV $334 \AA / F e \operatorname{xv} 284 \AA(\square)$, with the individual data points connected by a dotted line. The solid line is the first-order fit to the line-ratio temperature. The systematic error bars (inset), are from the relative intensity calibration and the uncertainties in atomic physics. Hence an error of the type shown would shift all data points from the same lineratio temperature in the same direction and by the same amount.

dependence of the line-ratio temperature for the rest of the analysis and is given in Table 2, in which the line-ratio temperatures are calculated using either the Arnaud \& Raymond (1992) or the Arnaud \& Rothenflug (1985) ionization balance. Since the SERTS 1989 southern edge had two Fe XIV spectral lines, the average temperature was used. The uncertainty of $T_{0}$ is dominated by the systematic uncertainty, while the uncertainty in $a$ is dominated by the uncertainty in the fit. $T_{e}$ was found to increase with height, to 1.15 $R_{\odot}$ for the SERTS 1989 data and to $1.20 R_{\odot}$ for the SERTS 1991 data, which were the maximum heights analyzed.

\subsubsection{Comparison of Different Ionization Balance Calculations}

Line-ratio temperatures determined by using the Arnaud $\&$ Raymond (1992) ionization balance are similar to those using Arnaud \& Rothenflug's (1985) values but with some systematic differences. The line-ratio temperatures (Table 2) determined by using the Arnaud \& Rothenflug (1985) ionization balance (as compared to the Arnaud \& Raymond

TABLE 2

LINE-RATIO TEMPERATURE

\begin{tabular}{|c|c|c|c|c|c|}
\hline \multirow[b]{2}{*}{ OBSERVATION } & \multirow[b]{2}{*}{$\begin{array}{l}\text { RANGE } \\
\left(R_{\odot}\right)\end{array}$} & \multicolumn{2}{|c|}{ Set $1^{\mathrm{a}}$} & \multicolumn{2}{|c|}{ Set $2^{b}$} \\
\hline & & $\begin{array}{c}T_{0}{ }^{\mathrm{c}} \\
\left(10^{6} \mathrm{~K}\right)\end{array}$ & $\begin{array}{c}a \\
\left(10^{6} \mathrm{~K}\right)\end{array}$ & $\begin{array}{c}T_{0}{ }^{\mathrm{c}} \\
\left(10^{6} \mathrm{~K}\right)\end{array}$ & $\begin{array}{c}a \\
\left(10^{6} \mathrm{~K}\right)\end{array}$ \\
\hline SERTS 1991 southern edge....... & $1.05-1.19$ & $1.90 \pm 0.14$ & $0.59 \pm 0.25$ & $1.87 \pm 0.13$ & $0.51 \pm 0.22$ \\
\hline SERTS 1989 southern edge...... & $1.02-1.15$ & $1.85+0.13$ & $0.70+0.19$ & $1.75+0.12$ & $0.71+0.30$ \\
\hline SERTS 1989 northern edge....... & $1.02-1.15$ & $2.02 \pm 0.17$ & $1.25 \pm 0.52$ & $1.97 \pm 0.15$ & $1.13 \pm 0.49$ \\
\hline
\end{tabular}


1992 ionization balance) were, on average, about $30,000 \mathrm{~K}$ cooler. In addition, the slope of the line-ratio temperature versus radius was smaller by $10 \%-20 \%$.

For the SERTS 1989 data sets, the results are similar to the data from SERTS 1991, except for the Fe XV/Fe XVI line-ratio temperature, which was not available from the SERTS 1991 data set. The Fe XV/Fe XVI line-ratio temperature decreased more than the other line-ratio temperatures when the Arnaud \& Rothenflug (1985) ionization balance was used instead of the Arnaud \& Raymond (1992) ionization balance (Table 2). The spread of line-ratio temperatures is reduced by using the Arnaud \& Rothenflug (1985) values. Hence, the line-ratio temperatures determined using the Arnaud \& Rothenflug (1985) ionization balance are more self-consistent. However, the uncertainties that are due to the possibility of temperature variation along the line of sight and in the measurements are large enough that the Arnaud \& Raymond (1991) ionization balance calculation cannot be ruled out.

\subsection{Emission Measure}

The emission measure can now be determined for each observed spectral line in the three data sets. The contribution function, $Q$, was evaluated using the least-square fit line-ratio temperatures given in Table 2 using line-ratio temperatures calculated with both sets of ionization balances. The contribution function was evaluated using the ionization balance of Arnaud \& Rothenflug (1985) for aluminum and silicon, that of Monsignori-Fossi \& Landini (1995) for chromium, and, for iron, the ionization balance used to calculate the line-ratio temperature.

The emission measures are plotted in Figure 12. The set of elemental abundances used come mainly from Meyer's (1985b) set of elemental abundances, except for chromium, for which Stone's (1989) elemental abundance was used, since Meyer did not provide the chromium abundance. The assumed relative elemental abundances are then $\mathrm{Fe}: \mathrm{Si}: \mathrm{Al}$ : Cr 1000: 1031: 90: 14.4. The emission measures at $1.05 R_{\odot}$ are given in Table 3, using the same set of elemental abun- dances. If Meyer's (1992, private communication) set of elemental abundances are used, the emission measures are divided by roughly 3.5 . The uncertainties used in Table 3 and Figure 12 were calculated using the uncertainty in the fit of the line-ratio temperature given in Table 2 and the instrument calibration relative uncertainty.

Emission measures for the SERTS 1989 data are plotted in Figures $12 b$ and $12 c$. The spread of the iron emission measures is less than the relative calibration uncertainties shown in the figures. The silicon emission measure from the SERTS 1989 southern edge agrees well with the iron emission measures.

For the SERTS 1991 southern edge, the emission measures determined for Fe XIII and Fe XIV (Fig. 12a) are in good agreement. The emission measure from $\mathrm{Fe}$ XVI was also in good agreement with the Fe XIII and Fe XIV emission measures above $1.08 R_{\odot}$, but it was larger than the others below $1.08 R_{\odot}$. This increase in the emission measure of $\mathrm{Fe}$ XVI close to the Sun is due to the hot loop (\$ 3.5.2), which emits weakly in the other, "cooler," spectral lines. The emission measures from spectral lines of other elements show the same general radial dependence as iron, but have different absolute values. This result will be discussed in greater detail in $\S 4.4$.

Calculations using the Arnaud \& Rothenflug (1985) ionization balance produced an iron emission measure $30 \%$ larger than calculations with the Arnaud \& Raymond (1992) values. Since the line-ratio temperatures calculated with these Arnaud \& Raymond (1992) values are systematically higher than temperatures obtained with the Arnaud \& Rothenflug (1985) ionization balance, the emission measures of the other elements decreased by $10 \%$, for a total change of roughly $40 \%$ for the Arnaud \& Raymond (1992) ionization balance as compared to the Arnaud \& Rothenflug (1985) ionization balance.

\subsection{Relative Elemental Abundances}

The most probable explanation for the SERTS 1991 southern edge having different emission measures for different elements is that the observed coronal elemental abun-

TABLE 3

EMISSION MEASURE AT $1.10 R_{\odot}^{\mathrm{a}}$

\begin{tabular}{|c|c|c|c|c|}
\hline Ion & $\begin{array}{l}\text { Wavelength } \\
(\AA)\end{array}$ & SERTS 1991 Southern Edge & SERTS 1989 Southern Edge & SERTS 1989 Northern Edge \\
\hline Fe xVI ...... & $\begin{array}{l}361 \\
335\end{array}$ & $\begin{array}{c}3.51(1.67) \\
\ldots\end{array}$ & $\begin{array}{c}1.55(1.68) \\
\ldots\end{array}$ & $1.35(1.44)$ \\
\hline $\mathrm{Fe} \mathrm{xv} \ldots \ldots$ & $\begin{array}{l}284 \\
417\end{array}$ & $\begin{array}{l}\ldots \\
\ldots\end{array}$ & $\begin{array}{c}5.97(1.95) \\
\ldots\end{array}$ & $\begin{array}{l}2.02(1.83) \\
1.47(1.80)\end{array}$ \\
\hline $\mathrm{Fe} \mathrm{xIV} \ldots \ldots$ & $\begin{array}{l}274 \\
334\end{array}$ & $3.86(1.17)$ & $\begin{array}{l}2.27(1.51) \\
1.37(1.54)\end{array}$ & $\begin{array}{c}0.86(1.32) \\
\ldots\end{array}$ \\
\hline $\begin{array}{l}\mathrm{Fe} \text { XIII ....... } \\
\text { Cr XIII ....... } \\
\text { Si XII ........ } \\
\text { Al x ....... }\end{array}$ & $\begin{array}{l}359 \\
328 \\
303 \\
332\end{array}$ & $\begin{array}{l}3.97(1.45) \\
4.55(1.46) \\
5.50(1.37) \\
17.2(1.51)\end{array}$ & $\begin{array}{c}\ldots \\
\ldots \\
0.60(1.29) \\
\ldots\end{array}$ & $\begin{array}{l}\cdots \\
\cdots \\
\cdots \\
\cdots\end{array}$ \\
\hline
\end{tabular}

${ }^{a}$ In units of $10^{27} \mathrm{~cm}^{-5}$. Errors are given in parentheses after values. The calculated errors are combinations of the instrument uncertainty and the uncertainty due to the line-ratio temperature. They do not include the absolute flux uncertainty of a factor of 2 . In addition, the ionization balance calculations and emissivity calculations could have a worst-case uncertainty as large as a factor of 2 , which is not included in the table. This latter uncertainty is not independent of the temperature uncertainties. Since the uncertainties in the temperature and in the ionization balance and the emissivity are interconnected, we can reasonably replace the errors in the above table with errors of a factor of 2 , which can then be viewed as an upper limit on the total uncertainty. All values are calculated using Arnaud \& Rothenflug's 1985 ionization balances. Using the elemental abundances of Meyer 1992 (private communication) gives values of the emission measures equal to those in the table divided by 3.5 . 


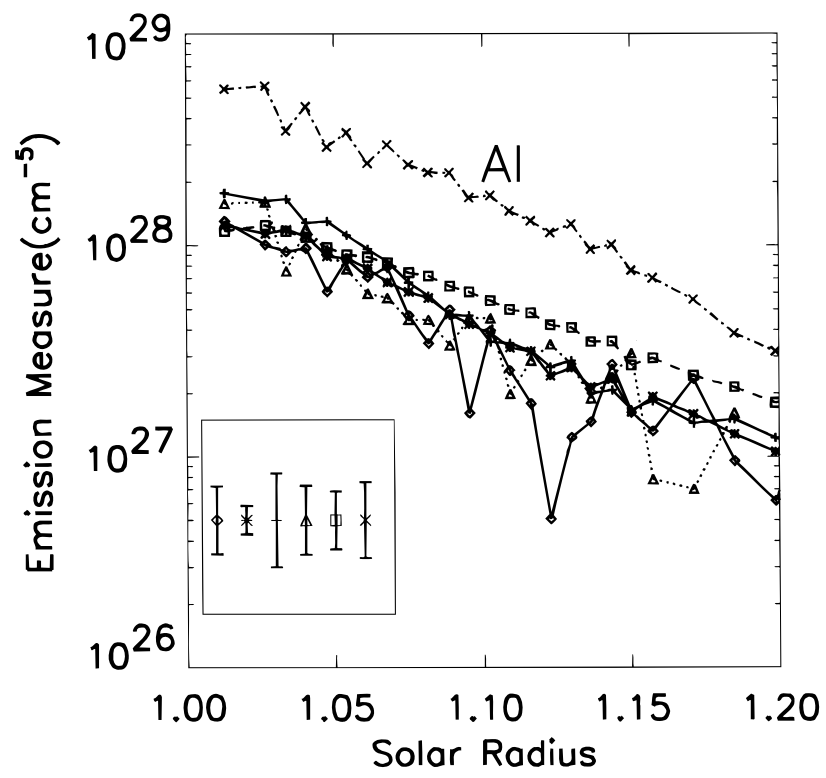

FIG. $12 a$

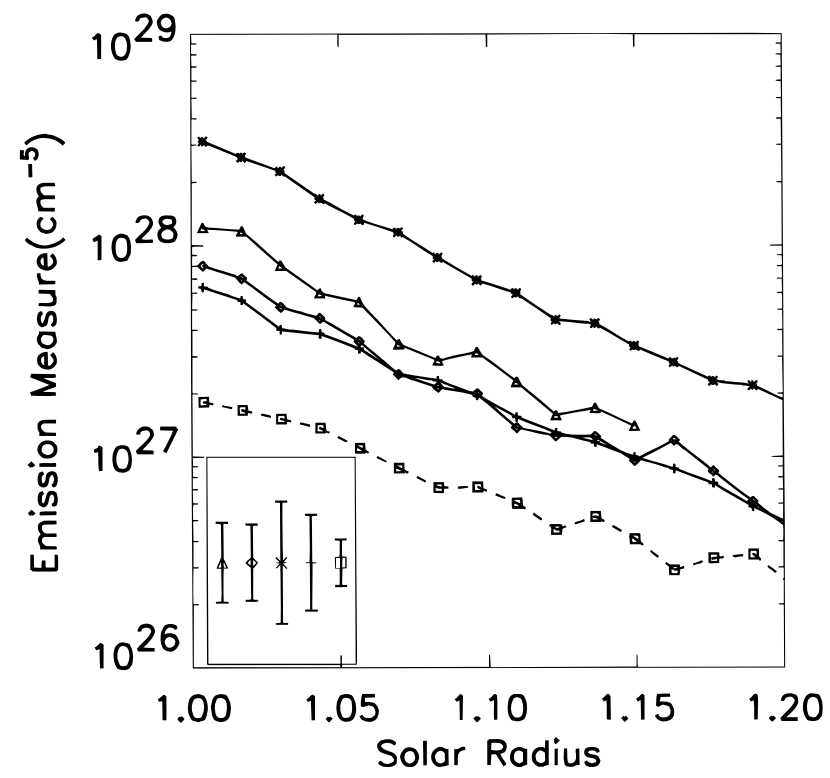

FIG. $12 b$

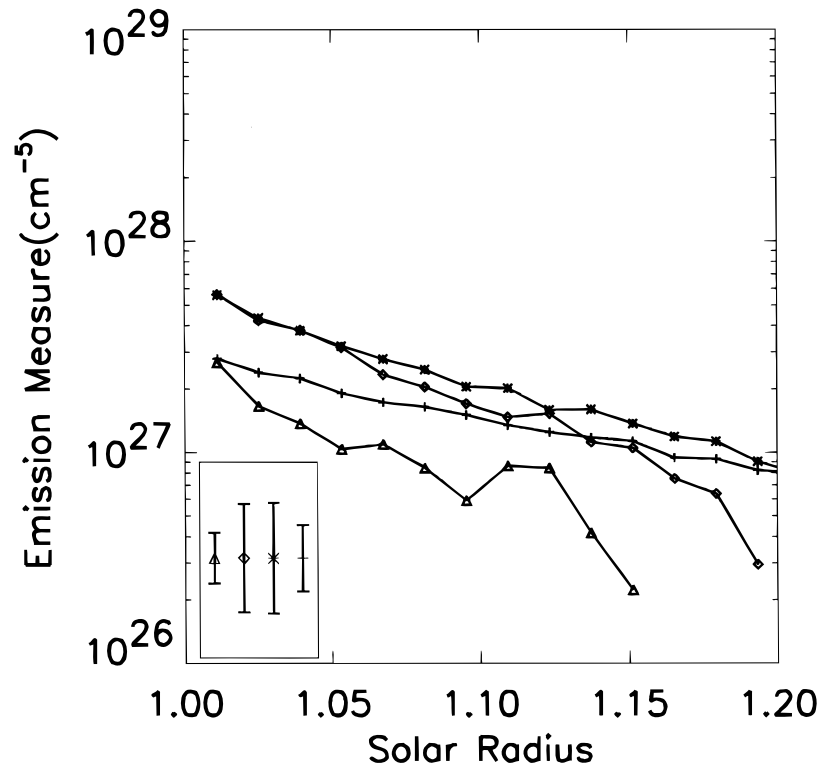

FIG. $12 c$

FIG. 12.-Emission measures as a function of radius for $(a)$ the SERTS 1991 southern edge, $(b)$ the SERTS 1989 southern edge, and $(c)$ the SERTS 1991 northern edge. The emission measures are calculated assuming Meyer's (1985b) elemental abundances and the line-ratio temperature given in Table 2, which in turn assumes Arnaud \& Rothenflug's (1985) ionization balance. The emission measures shown are (a) the Fe XVI $361 \AA(+), F e$ XIV $334 \AA(*), F e$ XIII 359.63 $\AA(\diamond), \mathrm{Cr}$ XIII $328 \AA(\triangle)$, Si XI $303 \AA(\square)$, and $\mathrm{Al}$ x $332 \AA(\times)$ lines, (b) the Fe XVI $361 \AA(+)$, Fe XV $284 \AA(*), F e$ XIV $334 \AA(\diamond), F e$ XIV $274 \AA(\triangle)$, and Si XI 303 $\AA(\square)$ lines, and $(c)$ the Fe XVI $361 \AA(+), F e x v 284 \AA(*), F e x v 417 \AA(\diamond)$, and Fe XIV $274 \AA(\triangle)$ lines. The error factor, defined in Table 3 , is shown in the inset box for each spectral line's emission measure, marked with the appropriate symbol. In $(a)$, the emission measure for Fe XVI is larger than for the other iron lines below $1.10 R_{\odot}$ because of the hot loop. The dips in the Fe xIII emission measure near $1.09 R_{\odot}$ and $1.12 R_{\odot}$ are probably due to the uncertainties in the background subtraction. Note that the emission measure for aluminum is consistently larger than for the other elements, which is explained by the difference between the observed plasma elemental abundance and Meyer's (1985b) elemental abundance.

dances for Al were different from those given by Meyer (1985b), which were used in deriving the emission measures. Dividing all the emission measures as a function of radius by the Fe XIV $334 \AA$ emission measure, we find the data shown in Figure $13 a$ (using line-ratio temperatures calculated using the Arnaud \& Rothenflug 1985 ionization balances) and Figure $14 a$ (using line-ratio temperatures calculated using the Arnaud \& Raymond 1992 ionization balances). In this representation, differences from the assumed set of elemental abundances show up as offsets. For the SERTS 1991 southern edge (Figs. 13a and 14a) the relative abundance of aluminum is high. The relative elemental abundances of chromium and aluminum are roughly constant with radius, while the elemental abundance of silicon appears to increase with radius. This later apparent increase is probably due to the increasing importance of resonance scattering with height for the Si XI line, rather than to a real change of relative elemental abundance with height. The observed relative elemental abundances are given in Table 4 and Figure 15 for line-ratio temperatures calculated with either the Arnaud \& Rothenflug (1985) or the Arnaud \& Raymond (1992) ionization bal- 


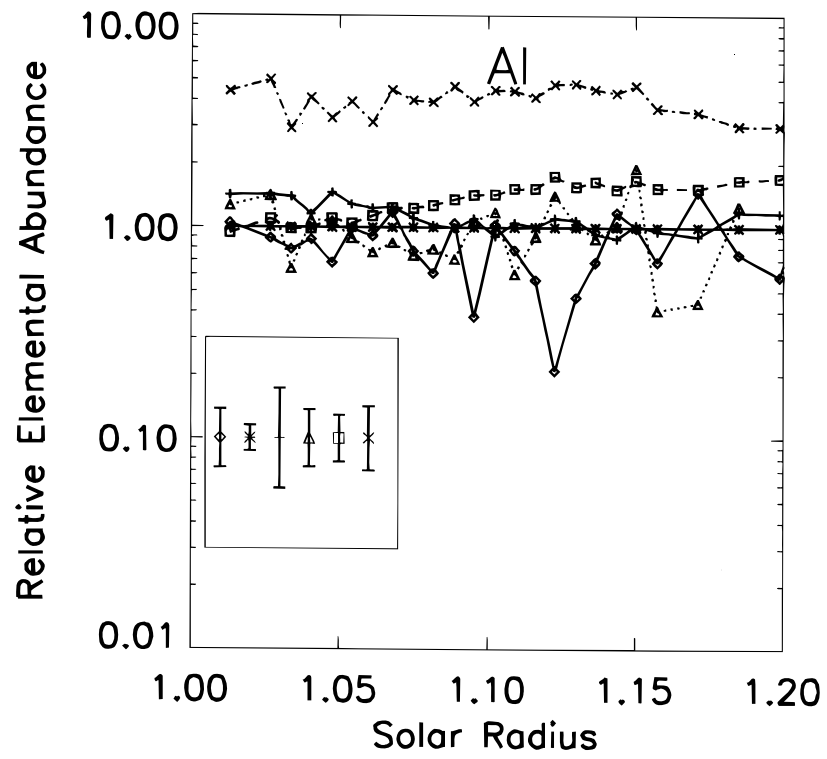

FIG. $13 a$

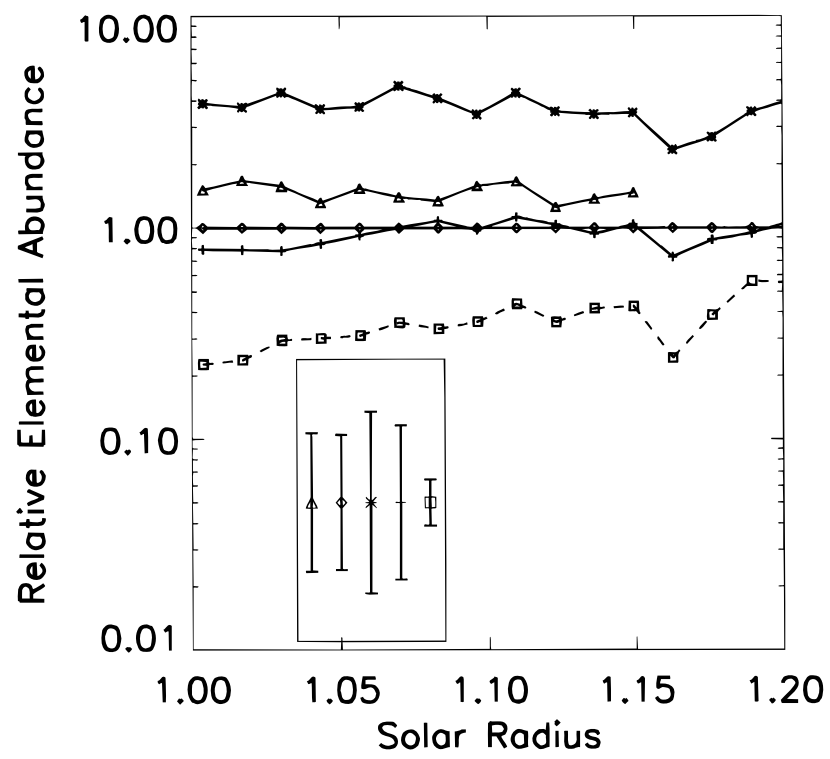

FIG. $13 b$

FIG. 13.- Observed relative elemental abundance relative to Meyer's (1985b) elemental abundance for $(a)$ the SERTS 1991 southern edge and (b) the SERTS 1989 southern edge. These plots use the emission measures shown in Fig. 12 divided by the emission measure of the Fe XIV $334 \AA$ spectral line, and are marked using the same symbols given in Fig. 12. Error bars are shown in the inset box. If the elemental abundances were the same as those of Meyer (1985), the relative elemental abundance for all spectral lines would be roughly 1 . For aluminum, it is consistently about 4 ; hence the aluminum elemental abundance is enhanced relative to the abundance of iron.

ances, and are compared with other published abundances for $\mathrm{Si}, \mathrm{Fe}, \mathrm{Cr}$, and $\mathrm{Al}$.

One possible explanation that can be ruled out for the apparent overabundance of aluminum in the SERTS 1991 flight is the presence of a large amount of cool $(\approx 1 \times$ $10^{6} \mathrm{~K}$ ) plasma along the line of sight. One problem with this explanation is that cool plasma would also cause excess emission of $\mathrm{Cr}$ XIII and Fe XIII spectral lines that is not seen. Also, the radial dependence of the emission measure would have to be the same as for the observed plasma. Some rough estimates to explain just the aluminum abundance would require an emission measure at $1 \times 10^{6} \mathrm{~K}$ approximately equal to that at $2 \times 10^{6} \mathrm{~K}$, which can be ruled out based on the low intensity in the other low-temperature spectral lines.

For the SERTS 1989 flight, only the relative abundance of silicon to iron can be determined because the lines from other elements were too weak to observe. The results are given in Figure $13 b$ using the line-ratio temperatures derived from Arnaud \& Raymond's (1992) ionization balances and in Figure $14 b$ using line-ratio temperatures

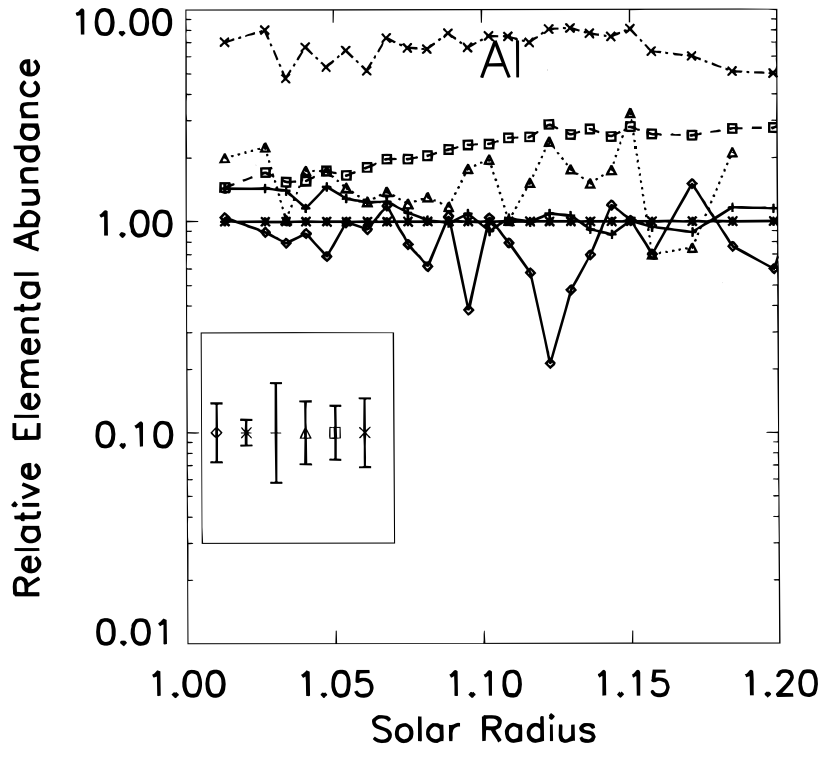

FIG. $14 a$

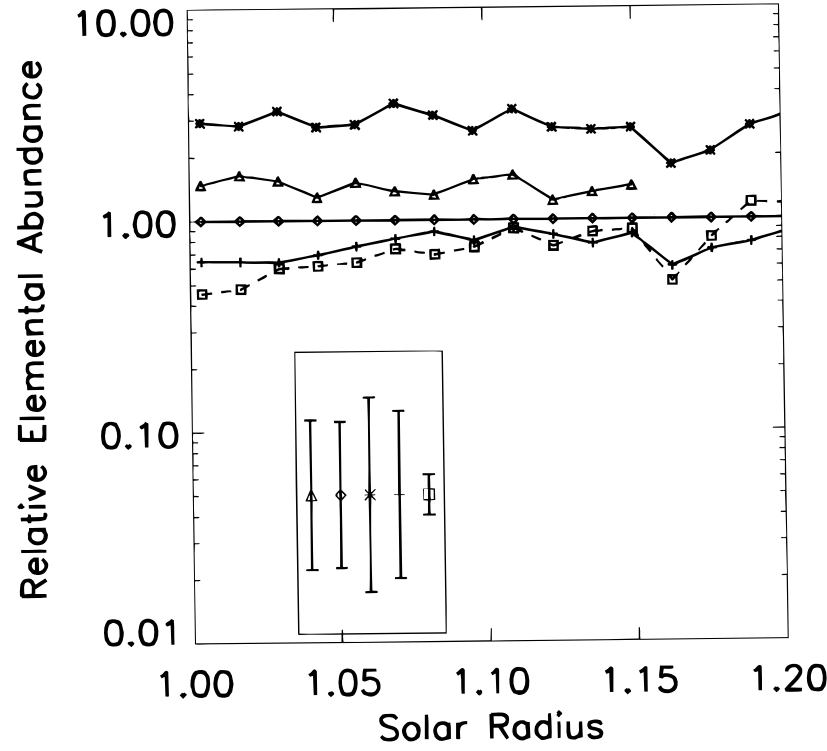

FIG. $14 b$

FIG. 14.- Same as Fig. 13, but using line-ratio temperatures calculated assuming Arnaud \& Raymond's (1992) ionization balances. The aluminum measured relative elemental abundance is consistently around 7 . 


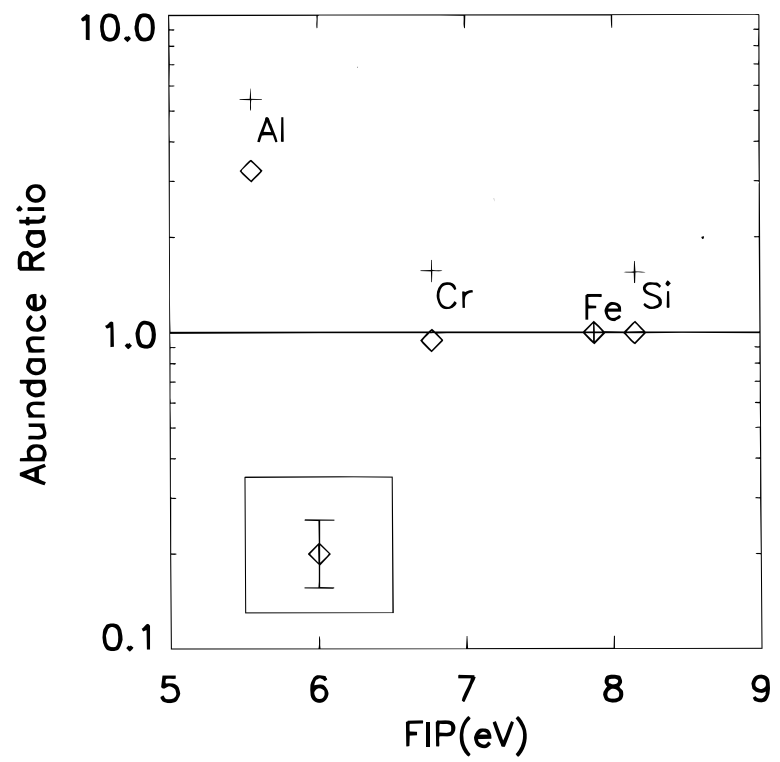

FIG. $15 a$

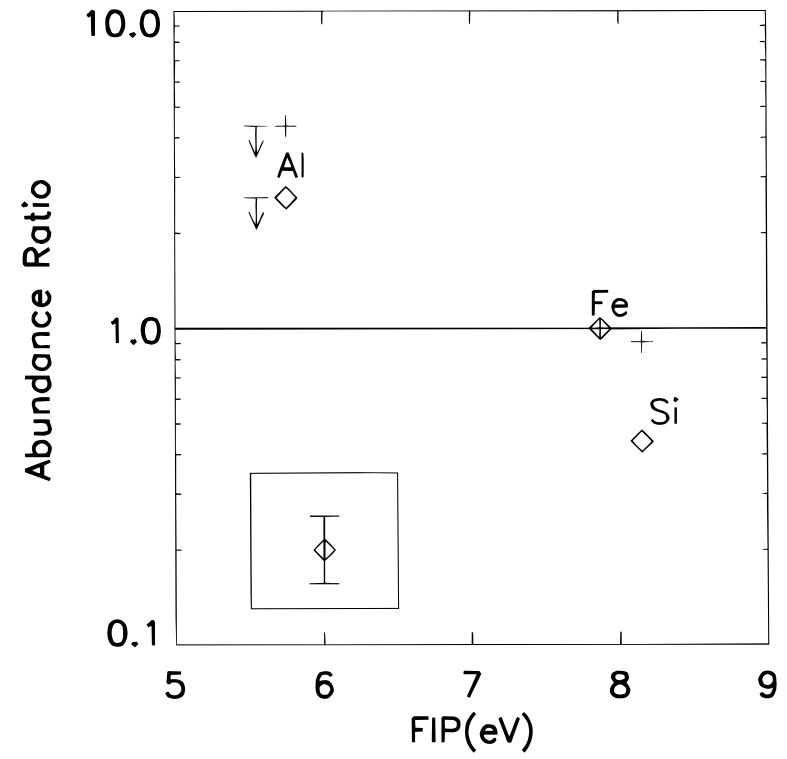

FIG. $15 b$

Fig. 15.- Ratio between the observed relative elemental abundance and the elemental abundances taken from Meyer (1985b), along with the chromium abundance from Stone (1989), where we take the abundance iron to be 1 . The observed relative elemental abundances calculated assuming either Arnaud \& Rothenflug's (1985) ionization balance (+) or Arnaud \& Raymond's (1992) ionization balance $(\diamond)$ are plotted for both the SERTS $1991(a)$ and the SERTS 1989 (b) southern sides. The error bars plotted in the lower left-hand corner are explained in Table 3. For the SERTS 1991 data (a) aluminum is overabundant, while for the SERTS 1989 data $(b)$ only the upper limit for the abundance of aluminum is plotted.

derived from Arnaud \& Rothenflug's (1985) ionization balances. The silicon-to-iron ratio agrees with Meyer (1985b).

An estimate of an upper limit of the relative abundance of aluminum to iron can be made for the SERTS 1989 southern edge. In Figure 16, SERTS 1989 spectra at $1.1 R_{\odot}$ are compared to the SERTS 1991 spectra scaled by the relative emission measures and taking into account the different line-ratio temperatures. The SERTS 1989 aluminum emission shows up at the $3 \sigma$ level of the background noise, shown as the solid horizontal line crossing the figure. The aluminum intensity from the 1989 data is $0.6 \pm 0.2$ of the scaled SERTS 1991 aluminum intensity. Part of the SERTS 1989 aluminum intensity probably comes from cooler emissions, which can be shown by noting that the SERTS 1989 emission in the 320-325 $\AA$ region is greater than the scaled SERTS 1991 intensity in the same region. The 320-325 region has lobes from $\mathrm{Si}$ VIII and $\mathrm{Mg}$ VIII, lines that have temperatures of maximum abundance at $800,000 \mathrm{~K}$. Plasma

TABLE 4

Relative Elemental Abundances

A. From SERTS 1991 AND 1989 DATA $^{\mathrm{a}}$

\begin{tabular}{|c|c|c|c|c|c|}
\hline \multirow[b]{2}{*}{ ELEMENTS } & \multirow{2}{*}{$\begin{array}{l}\text { FIP } \\
(\mathrm{eV})\end{array}$} & \multicolumn{2}{|c|}{$\mathrm{ARa}^{\mathrm{b}}{ }^{\mathrm{b}}$} & \multicolumn{2}{|c|}{ ARo85 } \\
\hline & & 1991 & 1989 & 1991 & 1989 \\
\hline Silicon $\ldots . . . \ldots \ldots$ & 8.15 & $2390(1.29)$ & $936(1.28)$ & $1250(1.41)$ & $453(1.25)$ \\
\hline Iron ................. & 7.87 & 1000 & 1000 & 1000 & 1000 \\
\hline Chromium ...... & 6.77 & $28.3(1.37)$ & $\ldots$ & $20.5(1.41)$ & $\ldots$ \\
\hline Aluminum ........ & 5.55 & $674(1.42)$ & $\cdots$ & $401(1.45)$ & $\cdots$ \\
\hline \multicolumn{6}{|c|}{ B. From the LiteratuRE } \\
\hline Elements & $\begin{array}{l}\text { FIP } \\
(\mathrm{eV})\end{array}$ & Allen (1973) & Meyer (1992) ${ }^{d}$ & Meyer (1985b) & Stone (1989) \\
\hline Silicon ........... & 8.15 & 832 & 1000 & 1031 & 787 \\
\hline Iron ............... & 7.87 & 1000 & 1000 & 1000 & 1000 \\
\hline Chromium $^{e}$...... & 6.77 & 17.8 & $\ldots$ & $\ldots$ & 14.4 \\
\hline Aluminum ......... & 5.55 & 62 & 70 & 90 & 66 \\
\hline
\end{tabular}

${ }^{a}$ Errors are given in parentheses after values. The errors are calculated as in Table 3.

${ }^{\text {b }}$ Using line-ratio temperatures calculated using Arnaud \& Raymond 1992 ionization balances.

${ }^{c}$ Using line-ratio temperatures calculated using Arnaud \& Rothenflug 1985 ionization balances.

${ }^{d}$ Meyer 1992, private communication.

${ }^{\mathrm{e}}$ Neither Meyer reference gives an abundance for chromium. 


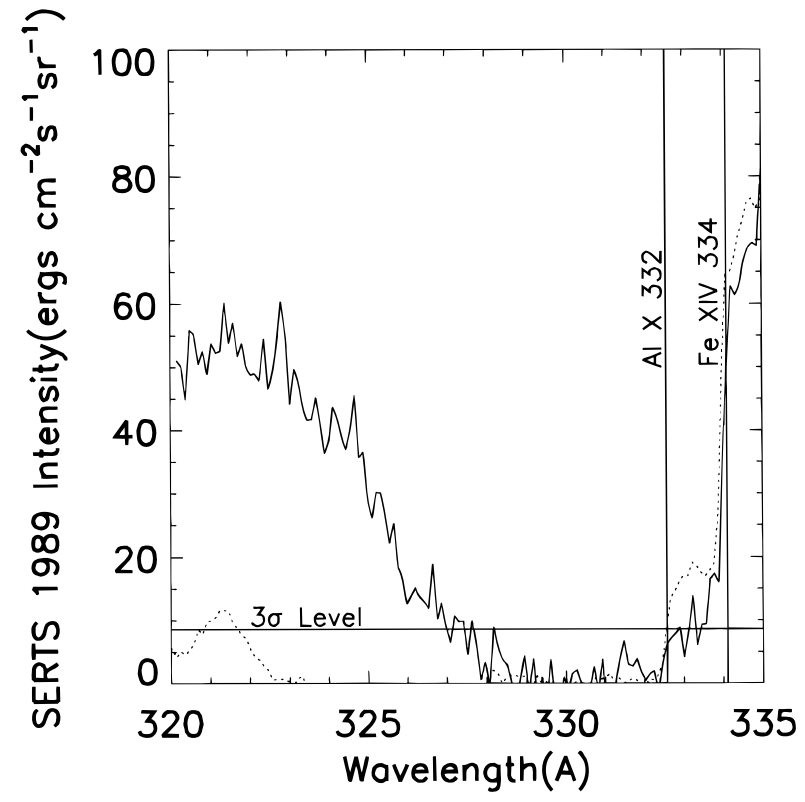

FIG. 16.-Solid line: $320-335 \AA$ spectrum after subtracting off the background between 330 and $331 \AA$ for the SERTS 1989 data. Dotted line: same, for the SERTS 1991 data, after dividing the intensity by 14 . The different scales are required by the differences in the emission measures and the effect of the line-ratio temperatures on the aluminum contribution function. The horizontal line indicates the $3 \sigma$ level detection above the background for a lobe nine $50 \times 50 \mu \mathrm{m}$ pixels wide. For the SERTS 1989 data, the Fe XIV $334 \AA$ spectral line is clearly above the $3 \sigma$ line and the Al x $332 \AA$ is just at the $3 \sigma$ line, while for the SERTS 1991 data both are detectable. at this temperature would also emit $\mathrm{Al}$ x $332 \AA$. Therefore, the SERTS 1989 southern edge aluminum abundance is probably even less than the upper limit $(0.6 \pm 0.2$ of the aluminum abundance determined for the SERTS 1991 data) determined from Figure 16.

\section{CONCLUSION}

We determined from SERTS 1991 observations of the quiet solar corona that the relative elemental abundance for the very low FIP element $\mathrm{Al}$ is enhanced compared to that of the low-FIP elements $\mathrm{Fe}, \mathrm{Si}$, and $\mathrm{Cr}$ by a factor of 3 or more (Fig. 15a). The degree of this enhancement can change, since the upper limit determined for the SERTS 1989 data (Fig. 14b) is less than the observed enhancement for the SERTS 1991 data. As mentioned earlier, Doschek et al. (1985) and Phillips \& Feldman (1991) reported that relative abundances of very low FIP elements can be enhanced compared to abundances of low-FIP elements in plumes and impulsive flares. This enhancement, we find, can also occur in plasma above the quiet Sun.

D. F. wishes to thank Professor George Goldenbaum, his dissertation advisor, and the members of his dissertation committee, Professor Mukul Kundu, Associate Professor Adil Hassam, Associate Professor Richard Ellis, and Senior Research Scientist Parvez Guzdar of the University of Maryland, and Joe Davila of GSFC/NASA. The research was supported by the NASA suborbital program and NASA grants 879-11-38 and 170-38-52.
Allen, C. W. 1973, Astrophysical Quantities (London: Athlone)

Arnaud, M., \& Raymond, J. 1992, ApJ, 398, 394

Arnaud, M., \& Rothenflug, R. 1985, A\&AS, 60, 427

Brickhouse, N. S., Raymond, J. C., \& Smith, B. W. 1995, ApJS, 97, 551

Brosius, J. W., et al. 1993, ApJ, 411, 310

Cook, J. W., Cheng, C.-C., Jacobs, V. L., \& Antiochos, S. K. 1989, ApJ, 338, 1176

Doschek, G. A., Feldman, U., \& Seely, J. F. 1985, MNRAS, 217, 317

Feldman, U. 1992, Phys. Scr., 46, 202

Feldman, U., \& Widing, K. G. 1990, ApJ, 363, 292

Fludra, A., \& Schmelz, J. T. 1995, ApJ, 447, 936

Fuhr, J. R., Martin, G. A., \& Wiese, W. L. 1988, J. Phys. Chem. Ref. Data, 17,194

Kelly, R. L. 1987, J. Phys. Chem. Ref. Data, 16, Suppl. 1, 1371

Martin, G. A., Fuhr, J. R., \& Wiese, W. L. 1988, J. Phys. Chem. Ref. Data, 17,370

McKenzie, D. L., \& Feldman, U. 1992, ApJ, 389, 764

Mewe, R., Gronenschild, E. H. M., \& van den Oord, G. H. J. 1985, A\&AS, 62, 197

Meyer, J. P. 1985a, ApJS, 57, 151 .1985b, ApJS, 57, 173

-.1993, Adv. Space Res. 13(9), 377

Monsignori-Fossi, B. C., \& Landini, M. 1995, in preparation (tables available at http://www.arcetri.astro.it)

Neupert, W. M., Epstein, G. L., Thomas, R. J., \& Thompson, W. T. 1992, Sol. Phys., 137, 87

\section{REFERENCES}

Phillips, K. J. H., \& Feldman, U. 1991, ApJ, 379, 401

Reames, D. V., Cane, H. V., \& von Rosenvinge, T. T. 1990, ApJ, 357, 259

Schmelz, J. T. 1993, ApJ, 408, 373

Sol.-Geophys. Data. 1989a, No. 538 (Boulder: Nat'l Geophysical Data Center)

- 1989b, No. 539 (Boulder: Nat'l Geophysical Data Center)

. 1991, No. 563 (Boulder: Nat'l Geophysical Data Center)

Stirling, A. C., Doschek, G. A., \& Feldman, U. 1993, ApJ, 404, 394

Stone, E. C. 1989, in AIP Conf. Proc. 183, Cosmic Abundance of Matter, ed. C. Jake Waddington (New York: AIP), 72

Strong, K. T., Lemen, J. R., \& Linford, G. A. 1991, Adv. Space Res., 11(1), 151

Thomas, R. J., Keski-Kuha, R. A. M., Neupert, W. M., Condor, C. E., \& Gum, J. S. 1991, Appl. Opt., 30, 2245

Thomas, R. J., \& Neupert, W. M. 1994, ApJS, 91, 461

Thompson, W. T., Neupert, W. M., Jordan, S. D., Jones, H. R., Thomas, R. J., \& Schmieder, B. 1993, Sol. Phys., 147, 29

Widing, K. G., \& Feldman, U. 1989, ApJ, 344, 1046

.1992, ApJ, 392, 715

Wiese, W. L., Smith, M. W., \& Miles, B. M. 1969, Atomic Transition Probabilities. Vol. II. Sodium through Calcium (Washington: Nat. Bur. Stand.)

Veck, N. J., \& Parkinson, J. H. 1981, MNRAS, 197, 41 